\title{
The effect of surface roughness on rotor-stator cavity flows
}

D. Fernando, S. Gao, and S. J. Garrett

Citation: Physics of Fluids 30, 064103 (2018); doi: 10.1063/1.5028209

View online: https://doi.org/10.1063/1.5028209

View Table of Contents: http://aip.scitation.org/toc/phf/30/6

Published by the American Institute of Physics

\section{Articles you may be interested in}

Extended integral wall-model for large-eddy simulations of compressible wall-bounded turbulent flows Physics of Fluids 30, 065106 (2018); 10.1063/1.5030859

Direct numerical simulation of quasi-equilibrium turbulent puffs in pipe flow

Physics of Fluids 30, 064102 (2018); 10.1063/1.5031884

Letter: Görtler-like vortices in an impinging shock wave/turbulent boundary layer interaction flow

Physics of Fluids 30, 061702 (2018); 10.1063/1.5034242

Bubble dynamics and atomization mechanisms in burning multi-component droplets

Physics of Fluids 30, 067101 (2018); 10.1063/1.5035384

Electroosmotic flow of Phan-Thien-Tanner fluids at high zeta potentials: An exact analytical solution Physics of Fluids 30, 062001 (2018); 10.1063/1.5033974

A new hybrid turbulence model applied to highly turbulent Taylor-Couette flow

Physics of Fluids 30, 065103 (2018); 10.1063/1.5025888

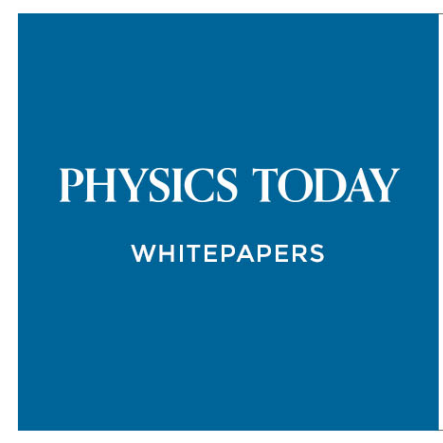

ADVANCED LIGHT CURE ADHESIVES

Take a closer look at what these environmentally friendly adhesive systems can do

\section{READ NOW}

PRESENTED BY

(8) MASTERBOND' 


\title{
The effect of surface roughness on rotor-stator cavity flows
}

\author{
D. Fernando, ${ }^{\text {a) }}$ S. Gao, ${ }^{\text {b) }}$ and S. J. Garrett ${ }^{\text {c) }}$ \\ Department of Engineering, University of Leicester, Leicester LE1 7RH, United Kingdom
}

(Received 8 March 2018; accepted 24 May 2018; published online 18 June 2018)

\begin{abstract}
We are concerned with the CFD simulation of annular rotor-stator cavities using the general purpose second-order finite volume method (FVM) solver OpenFOAM ${ }^{\circledR}$ and Large Eddy Simulation (LES) methods. Simulations of cavities with smooth surfaces are conducted at various Reynolds numbers, and the properties of the mean turbulent flows are validated against experimental and numerical data available in the literature. Comparisons show that second-order accurate FVM approaches can produce high-fidelity simulations of rotor-stator cavities to an acceptable accuracy and are therefore a viable alternative to the computationally intensive high-order methods. Our validated second-order FVM model is then combined with the parametric force approach of Busse and Sandham ["Parametric forcing approach to rough-wall turbulent channel flow," J. Fluid Mech. 712, 169-202 (2012)] to simulate cavities with a rough rotor surface. Detailed flow visualisations suggest that roughnessinduced disturbances propagate in the downstream direction of the rotor flow toward the outer wall of the cavity. The outer wall subsequently provides a passage to transport said roughness effects from the rough rotor layer to the smooth stator layer. We demonstrate that rotor-stator cavity flows are sensitive to even small roughness levels on the rotor surface alone. Published by AIP Publishing. https://doi.org/10.1063/1.5028209
\end{abstract}

\section{INTRODUCTION}

The flow inside a rotor-stator cavity is a topic of enduring interest within the scientific community, not only because of the rich and complicated flow structures found in its boundary layers but also owing to its relevance to many engineering applications. Common application areas include turbomachinery, the internal flows of combustion engines, mixing devices, computer storage and electronic devices, and machinery used for crystal growth processes.

In rotor-stator cavities of finite extent [see Fig. 1(a)], the flows over the rotor and stator elements are related but not identical to the Ekman ${ }^{1}$ and Bödewadt ${ }^{2}$ boundary layers. In particular, the lack of the radial pressure gradient causes a radial outflow within the rotor layer and a radial inflow on the stator layer, but shear layers are now observed on the outer sidewall (at $r_{2}$ ) and the inner rotor hub (at $r_{1}$ ) which permit an exchange of fluid between the rotor and stator layers. These shear layers arise, of course, from the finite nature of the cavity and lead to a deviation from the self-similar Ekman and Bödewadt solutions (over infinite disks) close to the sidewall. Investigations ${ }^{3}$ of the cylindrical cavity $\left(r_{1}=0\right)$ reveal that the self-similar velocity profiles apply at radial positions away from the outer wall with $r<0.87 r_{2}$.

Daily and $\mathrm{Nece}^{4}$ identified four flow regimes in cavities, the so-called regimes I-IV. Within their categorisation, the aspect ratio of the cavity, $G=h /\left(r_{2}-r_{1}\right)$, is the prime factor in determining whether the boundary layers of the upper and lower disks are merged (regimes I and III) or unmerged

\footnotetext{
a) bdrf1@ leicester.ac.uk

b) sg32@leicester.ac.uk

c) Author to whom correspondence should be addressed: sjg50@ leicester.ac.uk
}

(regimes II and IV). The Reynolds number, $R e_{\omega}$, (based on the rotation rate) then defines whether the flow is laminar (regime I and II) or turbulent (regime III and IV). In this current paper, all cavities are assumed to operate in regime II or IV. Furthermore, the cavities considered here are annular, that is, $r_{2}<\infty$ and $r_{1} \neq 0$. However, it is possible to extend our results to cylindrical cavities.

Previous studies of transition to turbulence within rotorstator cavities are typically based on experimental and numerical investigations. Sirivat ${ }^{5}$ conducted an experimental investigation of the stability of the stator boundary layer within a rotor-stator cavity under different aspect ratios $G=0.014$ -0.0475 and $R e_{h}$ values. Steady-state circular patterns were observed within the boundary layer for high $G$ values, but when the aspect ratios reduce to $G=0.014$, an alternative pattern appears with non-steady spiral patterns showing negative wave angles. These patterns are now known to correspond to stationary and travelling modes of the Type II instability that results from viscous effects including the streamline curvature. ${ }^{6}$ The alternative Type I instability, which always shows positive wave angles and results from inviscid crossflow effects, was detected at higher rotation speeds. Sirivat concluded that the disturbances emerge from the side walls and propagate inwards before collapsing. This then acts as the primary trigger for the appearance of turbulence. Gauthier et al. ${ }^{7}$ conducted additional experiments using a laser sheet visualisation technique. At a constant $G=0.048$, the circular instability patterns can be observed in the range of $70<R e_{h}<140$. In the higher Reynolds number region of $140<R e_{h}<200$, approximately 30 Type II spiral arms emerge, and eventually, beyond the Reynolds numbers of $R e_{h}>200$, turbulence spots appear. Subsequently, Schouveiler et al. ${ }^{8}$ constructed a detailed transition diagram that summarises the appearance of 

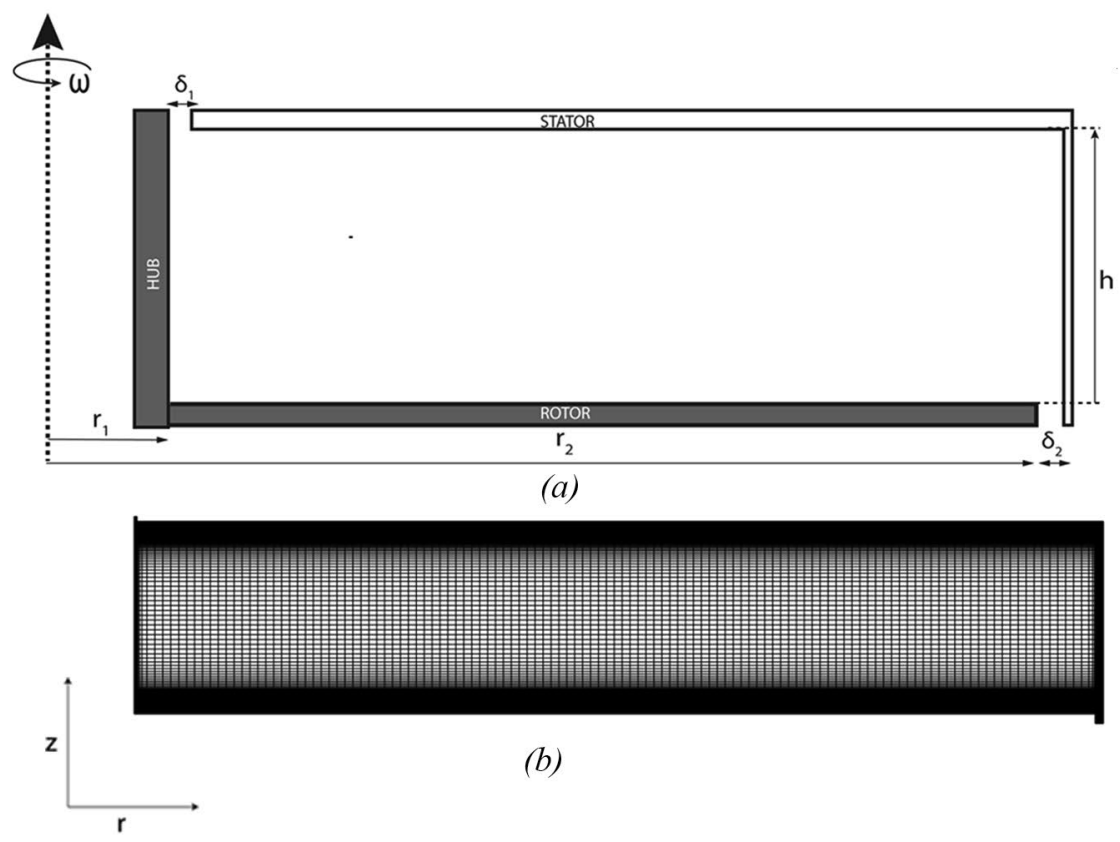

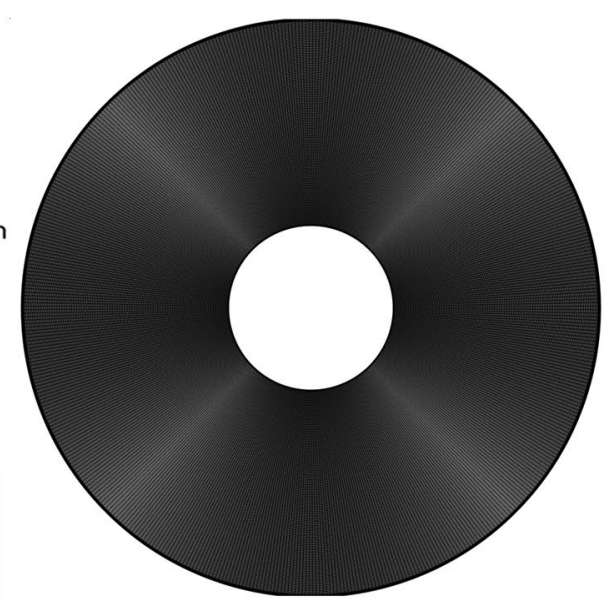

(c)

FIG. 1. The geometrical and mesh representations of the rotor-stator cavity: (a) side view of the finite rotor-stator cavity, (b) side view of the computational mesh, (c) top view of the computational mesh for $\operatorname{Re}_{\omega}=4 \times 10^{5}$.

all observed instability patterns (circular waves, spiral waves, solitary waves, and turbulent spots) at different combinations of Reynolds numbers and aspect ratios.

Serre et al. ${ }^{9}$ conducted DNS investigations of a cylindrical cavity $\left(r_{1}=0\right)$ with $G=0.2$ and 0.33 and an annular cavity $\left(r_{1} \neq 0\right)$ with $G=0.2$ and $R_{m}=\left(r_{2}+r_{1}\right) /\left(r_{2}-r_{1}\right)=4.5$. Observations of the stator boundary layer in both geometries were always consistent with those of previous experiments. However, eight travelling Type II waves were also observed in the rotor boundary layer for sufficient $R e_{\omega}$. More recently, Séverac et al. ${ }^{10}$ investigated a rotor-stator cavity with $G=0.2$ and $R_{m}=1.8$ using Large Eddy Simulation (LES) techniques for $R e_{\omega}=1 \times 10^{5}-1 \times 10^{6}$ and confirmed that the stator boundary layers become turbulent at much lower $R e_{\omega}$ values than the rotor boundary layers. At $R e_{\omega}=1 \times 10^{6}$, the rotor boundary layer consists of 19 spiral arms with an approximately $16^{\circ}$ wave angle within the region $0.14<r^{*}<0.61$; these are suggested to arise from the Type I instability. Either side of this region, the flow becomes turbulent as a likely result of the instability. Makino et al. ${ }^{11}$ conducted a similar numerical investigation and, at $R e_{\omega}=4 \times 10^{5}$, found the rotor boundary layer to feature 16 spiral arms. These were suggested to be due to a Type II instability - which is in contrast to the conclusions of Séverac et al.

So far, all investigations discussed have implicitly assumed smooth surfaces within the rotor-stator cavities, or that the effects of the roughness on the flow field are negligible. However, in general, there may be situations where the effects of roughness are no longer negligible. For example, Zoueshtiagh et al., ${ }^{12}$ and Harris et al., ${ }^{13}$ Cooper et al. ${ }^{14}$ and Garrett et al. ${ }^{15}$ conducted investigations into the transition of von Kármán-type boundary layers over rough rotating disks. All investigations agree that surface roughness has only a minor influence on the transition process up to a certain level of roughness, but beyond this, the flow is significantly stabilised via a reduction in the critical Reynolds number $R e_{c}$ for the onset of the dominant Type I mode. The latter studies suggest that roughness effects likely cause a switch in the dominant transition mechanism from the Type I mode to the Type II mode. Alveroglu et al. ${ }^{16}$ generalised the rotatingdisk studies to the full BEK family and studied the convective stability of these boundary layers against different types of roughness (radial grooves, concentric grooves, and isotropic roughness), finding consistent stabilising effects. The very recent investigation of Özkan et al. ${ }^{17}$ conducted experimental and numerical studies of rotor-stator cavities, paying particular attention to a comparative understanding of roughnessinduced and geometric-induced effects. They conclude that the geometric and roughness properties impose similar effects on the flow.

The content of this paper is twofold:

1. We determine the simulation conditions necessary to conduct rotor-stator cavity simulations using the open source CFD library OpenFOAM ${ }^{\circledR}$. This intends to demonstrate that second-order accurate FVM approaches can produce high-fidelity simulations of rotor-stator cavities to an acceptable accuracy. That is, this approach is a viable alternative to high-order methods for many different engineering rotor-stator flow simulations.

2. Using the parametric force model of Busse and Sandham, ${ }^{18}$ on the rotor surface in combination with our second-order accurate FVM LES approach, we investigate the effects of rough wall rotor-stator cavities.

\section{COMPUTATIONAL MODEL}

\section{A. Geometric model}

The general rotor-stator cavity considered here is illustrated in Fig. 1(a). It consists of two concentric disks rotating 
about a shared axis of rotation and with vertical spacing $h$. It is assumed that $h$ is large enough to separate the two boundary layers formed on the rotor (lower) and stator (upper) disks. The inner radius $r_{1}$ is the radius of the central hub wall (as measured from the axis of rotation) that is directly connected to the rotor. The outer radius $r_{2}$ is the distance between the axis of rotation and the periphery of the rotor where an outer wall is placed and fixed to the stator. The hub and outer wall are perpendicular to the rotor and stator. For an annular cavity geometry, $r_{1} \neq 0$, and the effective radial extent of the cavity is $\Delta r=r_{2}-r_{1}$. In the case that $r_{1}=0$, the cavity becomes cylindrical with radial extent $r_{2}$.

The operational Reynolds number based on the rotor periphery is taken as $R e_{\omega}=\frac{\omega r_{2}^{2}}{v}$, and the local Reynolds number at any given point $p$ on this rotor surface is $R e_{\omega, \text { local }}=\frac{\omega r^{2}}{v}$, where $r$ is the radial distance between point $p$ and the axis of rotation.

The physical assembly and machining of real rotor-stator cavities require finite gaps between rotating and stationary components of widths $\delta_{1}$ and $\delta_{2}$, as shown in Fig. 1(a). We capture this in our geometrical model to avoid any potential discrepancies with comparisons to experiments. The aspect ratio $G$ and curvature $R_{m}$, as defined in Eq. (1), are kept at the same constant values used in previous investigations, ${ }^{10,11}$

$$
G=\frac{h}{r_{2}-r_{1}}=0.2, \quad R_{m}=\frac{r_{2}+r_{1}}{r_{2}-r_{1}}=1.8 .
$$

The commercial mesh generation software ANSYS ICEM $\mathrm{CFD}^{\circledR}$ is used to generate a hexahedron computational mesh within the cavity. The number of elements used to construct the two configurations for the high and low Reynolds number cases is shown in Table I. Figures 1(b) and 1(c) show the side and top views of the computational mesh used for $R e_{\omega}=4 \times 10^{5}$ simulations.

\section{B. Numerical model}

The governing equations for the fluid flow within the cavity are the incompressible Navier-Stokes equations

$$
\begin{gathered}
\nabla \cdot \boldsymbol{u}=0, \\
\frac{\partial \boldsymbol{u}}{\partial t}+\boldsymbol{u} \cdot \nabla \boldsymbol{u}=-\nabla p+\nabla^{2} \cdot \boldsymbol{\tau}+\boldsymbol{F}_{\boldsymbol{b}},
\end{gathered}
$$

where $\boldsymbol{u}=\left(u_{x}, u_{y}, u_{z}\right), p$ is the specific pressure $\left(p=\frac{P}{\rho}\right)$, and $\left(\nabla=\frac{\partial}{\partial x}, \frac{\partial}{\partial y}, \frac{\partial}{\partial z}\right)$, all in Cartesian coordinates. The term $\boldsymbol{F}_{\boldsymbol{b}}$ is the specific force term, which bears the dimensions of acceleration $\left(L T^{-2}\right)$ and is such that $\boldsymbol{F}_{\boldsymbol{b}}=0$ for all smooth wall cases.

All simulations are conducted using the open source CFD library OpenFOAM which uses a second-order

TABLE I. The mesh configurations used in LES simulations.

\begin{tabular}{lcccc}
\hline \hline$R e_{\omega}$ & $r_{1}$ & $r_{2}$ & $\mathrm{~h}$ & $r \times \theta \times z$ \\
\hline $1 \times 10^{5}$ & 140 & 40 & 20 & $220 \times 180 \times 140$ \\
$4 \times 10^{5}$ & 280 & 80 & 40 & $256 \times 600 \times 180$ \\
\hline \hline
\end{tabular}

accurate FVM-based segregated solver. Our problem is therefore implemented using a collocated FVM approach ${ }^{19}$ to discretise Eqs. (2) and (3) and a second-order accurate central difference schema and implicit backward difference method to determine the convective and temporal integrals, respectively. The non-iterative PISO algorithm ${ }^{20}$ achieves the pressurevelocity coupling for solving the Navier-Stokes equations. The no-slip boundary condition applies at all walls, and the rotating motion of the rotating disk is set through

$$
\boldsymbol{u}_{\text {rotor }}=\omega\left(\boldsymbol{r}-\boldsymbol{r}_{\text {centre }}\right) \times \boldsymbol{I}_{\text {axial }} \text {. }
$$

Here $\boldsymbol{r}$ and $\boldsymbol{r}_{\text {centre }}$ are the position vectors to a given point on the rotor surface and the position vector of the centre of the disk, respectively, and $\boldsymbol{I}_{\text {axial }}=(0,0,1)$ is the axial directional vector. Operational Reynolds numbers are achieved by adjusting the rotation rate $\omega$.

The turbulent stresses in Eq. (3) are resolved through the LES approach. The sub-grid scale model is similar to the Smagorinsky ${ }^{21}$ model, but the model constants are determined by following the systematic dynamic procedure suggested by Germano et al. ${ }^{22}$ In particular, the scale separation of the LES method is usually done through convoluting the Navier-Stokes equations [Eqs. (2) and (3)] with a filter kernel, $K\left(\boldsymbol{r}, \Delta_{g}\right)$, associated with the position vector $\boldsymbol{r}$ and the filter width $\Delta_{g}$. However, in our simulation, a top-hat kernel with a grid-based filter width $\Delta_{g}=\sqrt[3]{\delta x \delta y \delta z}$ has been used. Furthermore, our dynamic Smagorinsky approach uses a test filter width $\overline{\Delta_{t}}>\Delta_{g}$ (usually $\overline{\Delta_{t}} \approx 2 \Delta_{g}$ ) to refine test scale stresses, which indeed are used to estimate the sub-grid scale stresses $\tau_{\text {sgs }}$. The implementation of the model used here is described by Fureby et al., ${ }^{23}$ (see model B) and the interested reader is referred there for full details.

As mentioned by Inagaki et al., ${ }^{24}$ the dynamic Smagorinsky model has been claimed to be less accurate than the standard Smagorinsky model or the mixed-time scale sub-grid scale model which was used in the previous investigation of Makino et al. However, both of these models are static models that require heuristic inputs to operate; such inputs are very much application specific and their determination for the flow conditions likely to be present in rotor-stator cavities is prohibitively complicated. The degree of inaccuracy of dynamic Smagorinsky models is often attributed to the averaging process used in the homogeneous direction ${ }^{24}$ which is important to achieve stable longer integration times for simulations. In particular, the general purpose dynamic Smagorinsky model implemented in OpenFOAM provides stable solutions over a substantial integration time, but model constants no longer represent the true local behavior due to the averaging process over the entire domain. Global model constants are not ideal in our situation as we expect locally laminar, transitional, and turbulent regions in the cavity. As a solution, we follow a local smoothing technique by refining the averaging operation over the neighbouring cells of the mesh. This process delivers a spectrum of model constants, each based on the local conditions of the rotor-stator cavity. This practice is expected to discount the disadvantages commonly linked to dynamic Smagorinsky models and reap the benefit of a simplistic sub-grid scale model which can adapt according to local flow conditions. 


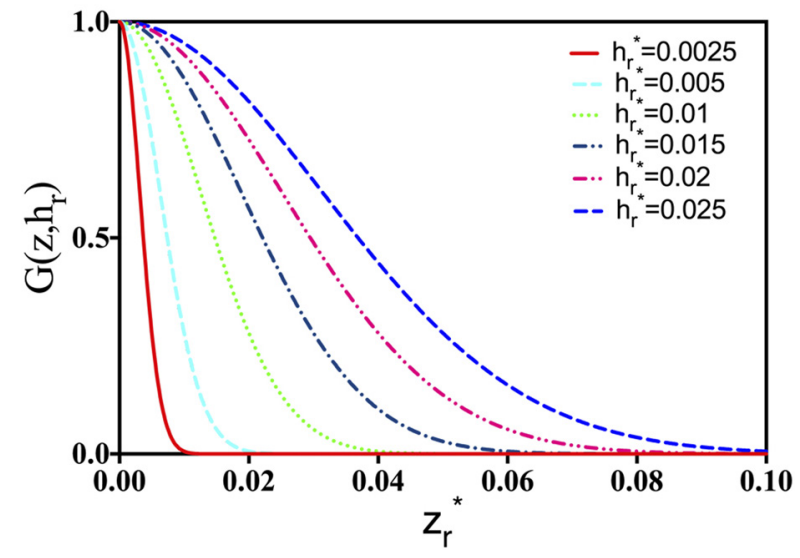

FIG. 2. The variation of the extent of numerical roughness layers at different $h_{r}^{*}$ values.

The dynamic Smagorinsky model with this modification allows the systematic calculation of the model constants, which represent the local behavior of the flow field. We deliberately increased the mesh density near the wall areas [see Fig. 1(b)], so there is no need to implement any damping function near the wall areas, and, in all the cases, the near wall resolution is maintained at $z_{\max }^{+}<1.0$. The time step size is based on the Courant number $(\mathrm{Co})$ that is limited to Co $<0.325$ in our simulations.

\section{Roughness model}

Two possible approaches can be taken for simulating the rough wall: direct simulation methods ${ }^{25-30}$ and roughness modeling methods. ${ }^{18,31-35}$ Direct simulation methods are the most precise; however, due to their significant computing requirements, they are more suited to simple geometric configurations. In the case of the rotor-stator cavities of interest here, direct methods impose a significant computational burden due to the increased number of mesh elements required and the rotation near the rotor boundary. Roughness modeling methods are viable alternatives for such applications. In fact, recent investigations of Chung et al. ${ }^{36}$ and Ishida et al. ${ }^{37}$ have shown that the roughness modeling methods coupled with high-fidelity numerical simulations, such as DNS or LES, could deliver results comparable to direct roughness simulations. Nevertheless, roughness models typically require the use of a thin numerical roughness layer near the wall surface, within which the roughness parameters are defined. Hence, these roughness models are not suitable for studying the physics and dynamics of flow in the vicinity of the physical roughness element- this often limits these models to the study of $k$-type roughnesses (as defined by Ref. 38).

The parametric force model of Busse and Sandham ${ }^{18}$ has been chosen to model the rough rotor wall. The approach has the advantage that it can represent $k$-type roughness as a body force. In particular, we use a body force with the $i$ th component given by

$$
F_{i}=-\alpha_{i} G\left(z, h_{r}\right)\left(u_{i}-u_{d, i}\right)\left|u_{i}-u_{d, i}\right|,
$$

where $i=1,2,3$ denotes the stream-wise, span-wise, and wall normal directions. Furthermore, $\alpha_{i}, G\left(z, h_{r}\right), h_{r}, u_{i}$, and $u_{d, i}$ are the roughness factor, shape function, roughness height, local flow velocity, and local disk surface velocity, respectively. The difference between the disk velocity and local fluid velocity at the disk surface implies the local relative velocity. The quadratic term in the right-hand side of Eq. (5), $\sim\left(u_{i}-u_{d, i}\right)$ $\left|u_{i}-u_{d, i}\right|$, emulates drag effects arising from the rough wall. Moreover, the roughness factor, $\alpha$, is interpreted as the linear density of roughness elements. Different $\alpha$ values can be associated with different roughness heights, $h_{r}$, to simulate many types of surface conditions. In Secs. III and IV, both modeling parameters, $\alpha$ and $h_{r}$, are presented as normalised against the height of the cavity (disk separation) $h$ so that one can compare the effects of roughness regardless of the cavity height.

For modeling purposes, the roughness factor is assumed to be $\alpha_{i}=\beta_{1} / k_{r}$, where $k_{r}$ and $\beta_{1}$ are the height of the physical roughness element and a proportionality constant, respectively. For a roughness element with $k_{r}=100 \mu \mathrm{m}$, the range $\beta_{1}=5 \times 10^{-4}$ to $5 \times 10^{-3}$ gives non-dimensioned roughness factors of $\alpha^{*}=h \alpha=0.1-1.0$. This range is sufficient to simulate wall roughness effects on the flow field and is free from any shielding effects on the rough wall. ${ }^{38}$

The shape function defines the extent of the roughness force in the wall normal direction and is usually associated with $h_{r}$. Busse and Sandham ${ }^{18}$ have defined several options for shape functions, the simplest being the box profile. ${ }^{36}$ However, the discontinuity near the top of the box profile tends to

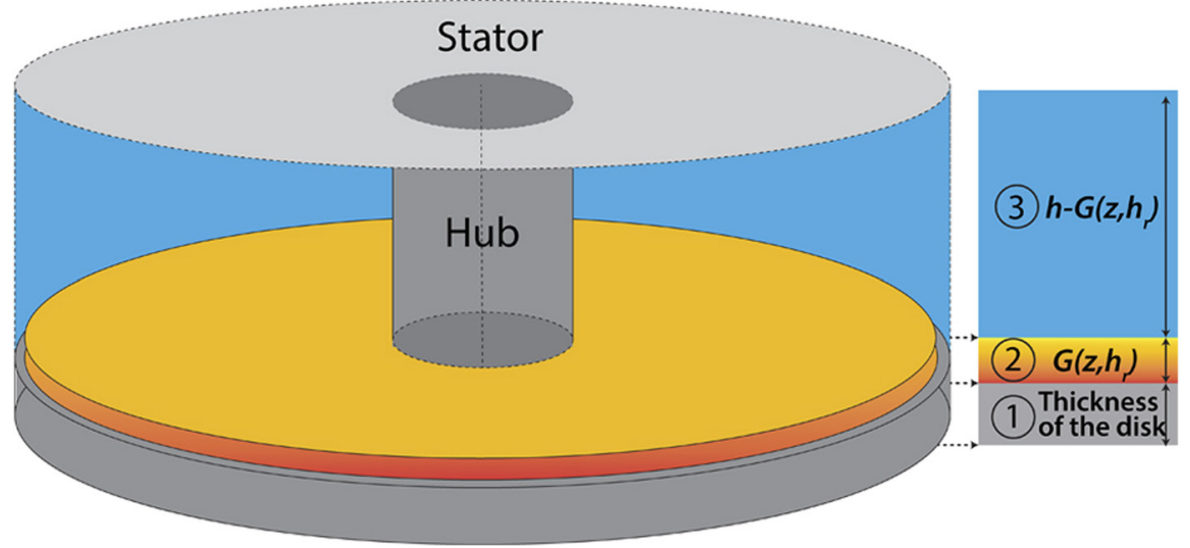

FIG. 3. An illustration of the different numerical regions arising from the use of the parametric model to impose surface roughness on the lower rotor. 
TABLE II. The modeling and geometric parameters used in simulations. Configurations with $\alpha^{*}=0$ or $h_{r}^{*}=0$ represent smooth wall cases.

\begin{tabular}{ccccc}
\hline \hline$R e_{\omega}$ & $\alpha^{*}$ & $h_{r}^{*}$ & Shape function & $\mathrm{h}(\mathrm{mm})$ \\
\hline $1 \times 10^{5}$ & $0-1.0$ & $0-0.025$ & Gaussian & 20 \\
$4 \times 10^{5}$ & $0,0.4$ & $0,0.00125,0.00375$ & Gaussian & 40 \\
\hline
\end{tabular}

introduce abrupt inflection points in the radial and tangential velocities. Gaussian shape functions, which can be considered as equivalent to "smeared-out" box profiles, ${ }^{18}$ provide considerably smoother velocity profiles and are the preferred choice. Here, the values of the $h_{r}$ act as a mean roughness height, and Eq. (6) determines the exact roughness-affected region.
The effects of $h_{r}$ are introduced through another auxiliary parameter, $\eta\left(h_{r}\right)$, as defined in Eq. (7),

$$
\begin{gathered}
G\left(z, h_{r}\right)=e^{-z^{2} / \eta\left(h_{r}\right)^{2}}, \\
\eta\left(h_{r}\right)=h_{r} \sqrt{\pi} .
\end{gathered}
$$

The current investigation accommodates a range of $h_{r}^{*}=\frac{h_{r}}{h}$ values to evaluate the effects of roughness height on the flow characteristics. Usually, the roughness height and physical roughness height relate as $h_{r} \propto k_{r}$. Figure 2 shows the variation of $G\left(z, h_{r}\right)$ under the selected value range $h_{r}^{*}=2.5 \times 10^{-3}$ to $2.5 \times 10^{-2}$. As shown in Fig. 3, the physical implementation of the parametric force model divides the domain into three regions.

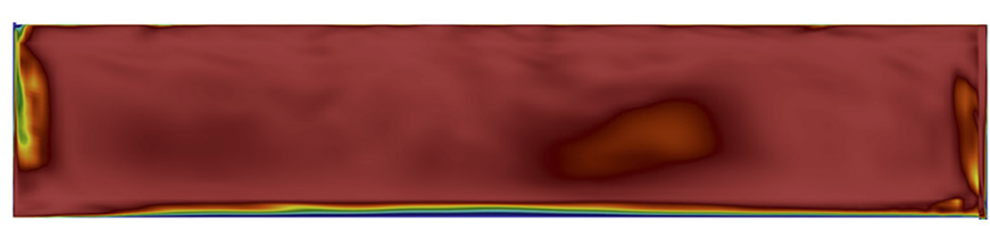

(a)

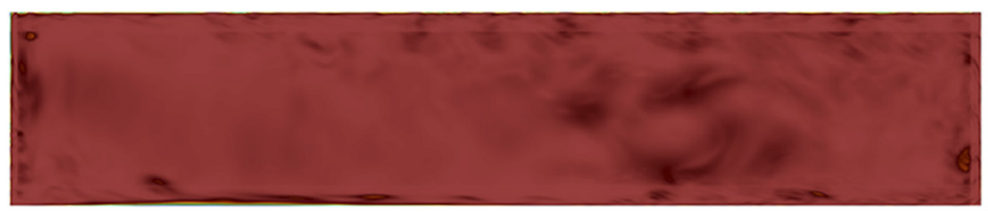

(b)

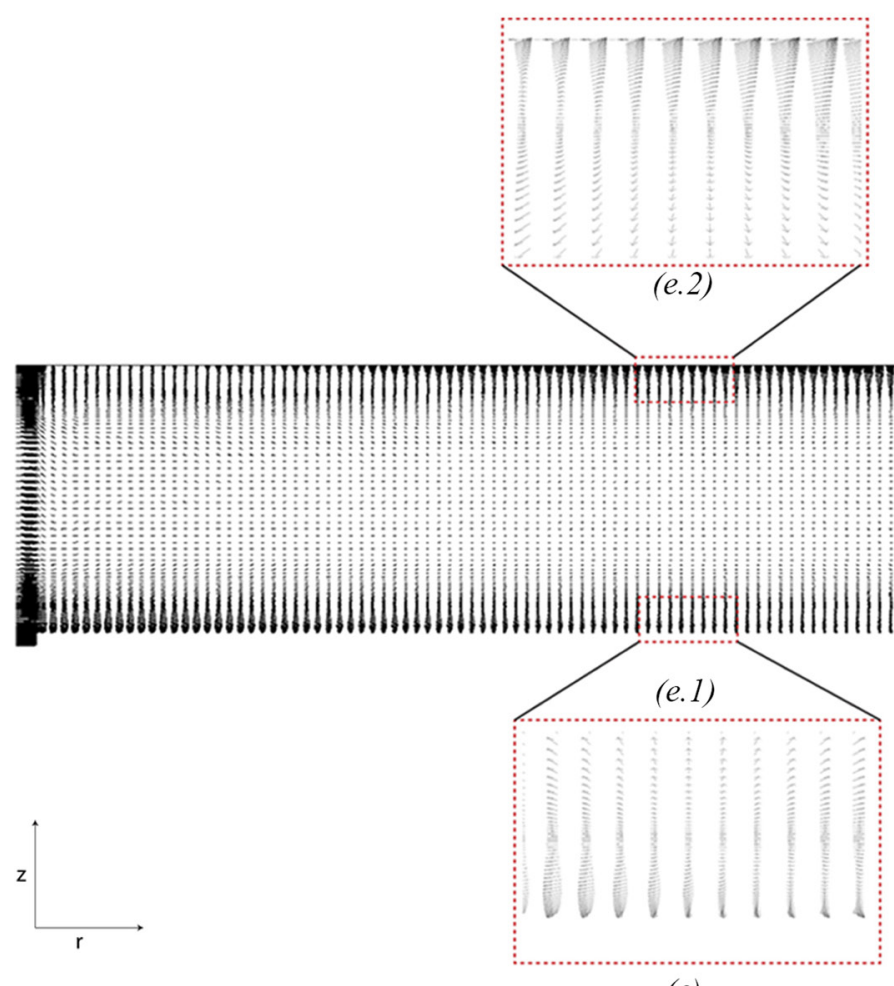

(e)
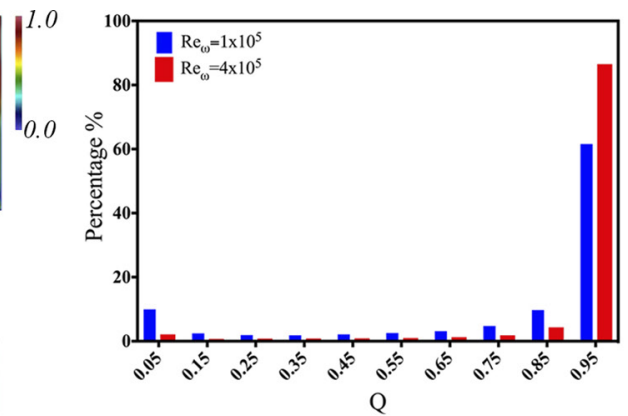

(c)

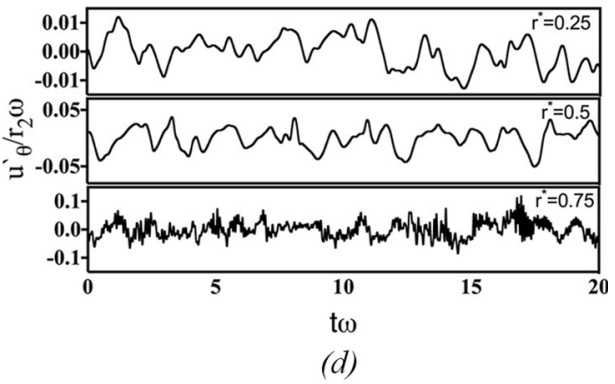

(d)

FIG. 4. (a) Contours of $Q$ for $R e_{\omega}=1 \times 10^{5}$, (b) contours of $Q$ for $R e_{\omega}=4 \times 10^{5}$, (c) $Q$ versus percentage of cells, (d) time series of the normalised tangential fluctuation component, $u_{\theta}^{\prime}$, at radial $r^{*}=0.25,0.50$, and 0.75 and axial $z^{*}=0.0125$ locations, (e) vector plot of the smooth wall rotor-stator cavity in the $r-z$ plane, $(e .1)$ at the rotor boundary and (e.2) at stator boundary. 
1. The disk surface (gray region): here the rotating boundary conditions are set according to Eq. (4).

2. Numerical roughness layer (yellow region): here the nonzero force is imposed, with Eq. (6) determining the size and the extent of the force.

3. Outside the roughness layer (blue region): here the force in Eq. (6) is zero, and the governing equations are equivalent to the smooth case.
Table II summarises all modeling and geometric configurations that will be used in Sec. III for the production of our results.

\section{RESULTS}

\section{A. Smooth rotor-stator cavity}

This section discusses the validation of our model against smooth rotor-stator cavities with $R e_{\omega}=1 \times 10^{5}$ and $4 \times 10^{5}$.

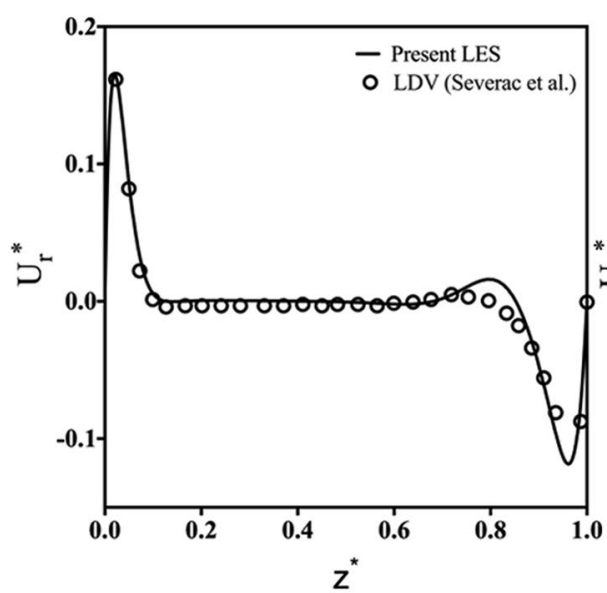

(a)

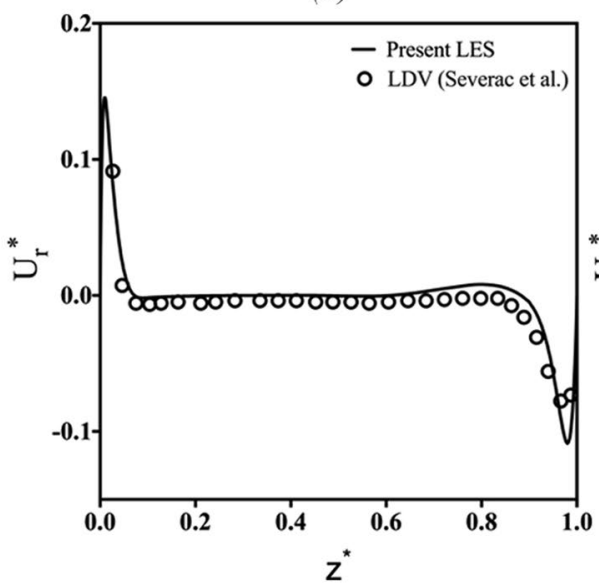

(c)

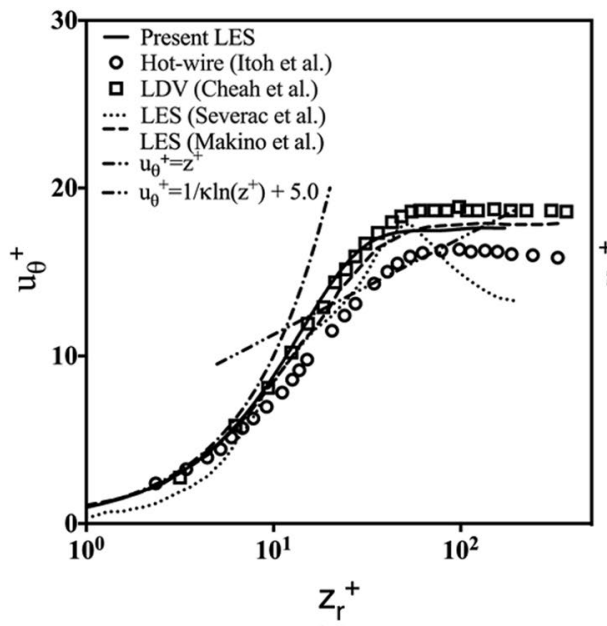

(a)

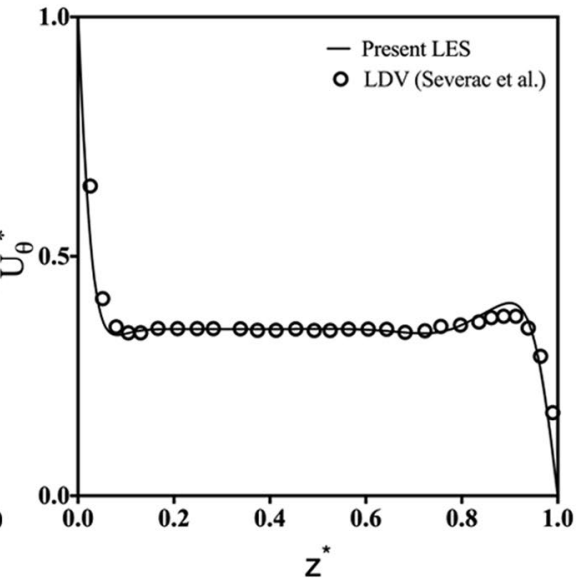

(b)

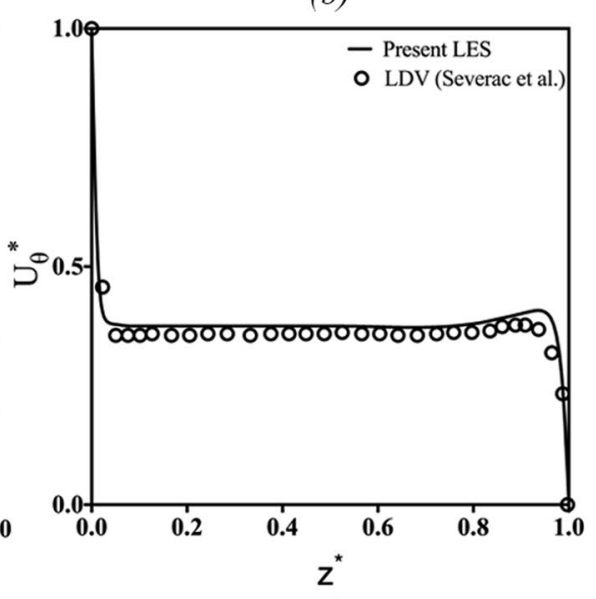

(d)

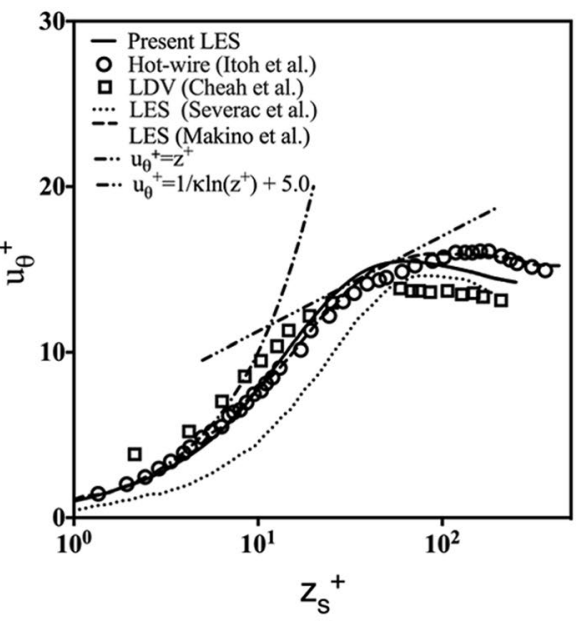

(b)
FIG. 5. The axial distributions of mean velocity fields for smooth cavities: (a) radial velocity profiles at $R e_{\omega}=1 \times 10^{5}$, (b) tangential velocity profiles at $R e_{\omega}=1 \times 10^{5}$, (c) radial velocity profiles at $R e_{\omega}=4 \times 10^{5}$, and (d) tangential velocity profiles at $R e_{\omega}=4 \times 10^{5}$.

FIG. 6. The mean tangential velocity profiles in the turbulent boundary layer at $R e_{\omega}=4 \times 10^{5}$ for smooth cavities: (a) rotor boundary layer and (b) stator boundary layer. Note that subscripts on the spatial variables indicate distances from the rotor or stator. 
TABLE III. A comparison of different configurations of smooth rotor-stator cavities in previous numerical and experimental investigations and the fitted $\kappa$ and $B$ values from the model in Eq. (8) for smooth turbulent rotor and stator boundary layers.

\begin{tabular}{|c|c|c|c|c|c|c|c|c|}
\hline \multirow[b]{2}{*}{ Investigation } & \multirow[b]{2}{*}{ Method } & \multirow[b]{2}{*}{$R e_{\omega, \text { local }}$} & \multirow[b]{2}{*}{ G } & \multirow[b]{2}{*}{$R_{m}$} & \multicolumn{2}{|c|}{ Rotor } & \multicolumn{2}{|c|}{ Stator } \\
\hline & & & & & $\kappa^{-1}$ & $B$ & $\kappa^{-1}$ & $B$ \\
\hline Present & LES & $2.2 \times 10^{5}$ & 0.2 & 1.8 & 14.9 & -5.5 & 13.5 & -5.6 \\
\hline Itoh et al..$^{43}$ & Hot-wire & $3.6 \times 10^{5}$ & 0.08 & 1.0 & 12.7 & -5.2 & 11.3 & -3.7 \\
\hline Cheah et al..$^{40}$ & LDV & $1.9 \times 10^{5}$ & 0.12 & 1.0 & 15.4 & -6.5 & 9.4 & -0.1 \\
\hline Makino et al. ${ }^{11}$ & LES & $2.3 \times 10^{5}$ & 0.2 & 1.8 & 12.4 & -3.6 & 12.5 & -4.8 \\
\hline Séverac et al. ${ }^{10}$ & LES & $4.1 \times 10^{5}$ & 0.2 & 1.8 & 14.0 & -5.5 & 15.0 & -10.8 \\
\hline
\end{tabular}

As discussed in Sec. II B, resolution and the quality of the mesh are an important factor for a successful LES approach. A finer mesh can directly resolve higher frequency structures, and the finest mesh can resolve the smallest structures of the problem, i.e., the Kolmogorov scales, in which LES is almost in line with DNS (see Ref. 39). However, this practice is not always possible, as the resolution of the mesh is often limited by the available computing resources. The configurations and resolutions of meshes are carefully selected to simulate rotor-stator cavities, and we define a parameter, $Q=k / k_{\text {total }}$, to evaluate the adequacy of the mesh resolution. Here, $k_{\text {total }}=k+k_{\text {sgs }}$ is the total turbulent kinetic energy and $k=0.5\left(\overline{u_{r}^{\prime} u_{r}^{\prime}}+\overline{u_{\theta}^{\prime} u_{\theta}^{\prime}}+\overline{u_{z}^{\prime} u_{z}^{\prime}}\right)$ and $k_{s g s}=1 / 2 \operatorname{tr}\left(\tau_{\text {sgs }}\right)$ are the resolved and sub-grid scale components of the turbulent kinetic energy, with $\operatorname{tr}(\cdot)$ giving the trace of a tensor. The LES converges to DNS when $Q \rightarrow 1.0$. Figures 4(a) and 4(b) show contours of $Q$ in the midsection of the $r-z$ plane, and in both $R e_{\omega}=1 \times 10^{5}$ and $4 \times 10^{5}$ cases, the most of the regions in the cross section occupy $Q$ values very close to unity. Figure 4(c) presents the percentage of cells versus the $Q$ value. The histogram was constructed by considering the entire simulation domain and shows that a high percentage of cells in the simulations is $Q>0.95$ for both the lower and higher $R e_{\omega}$ cases.

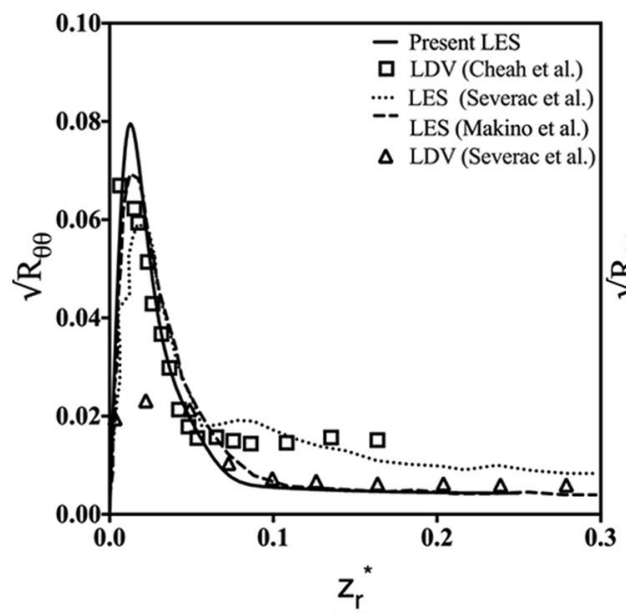

(a)

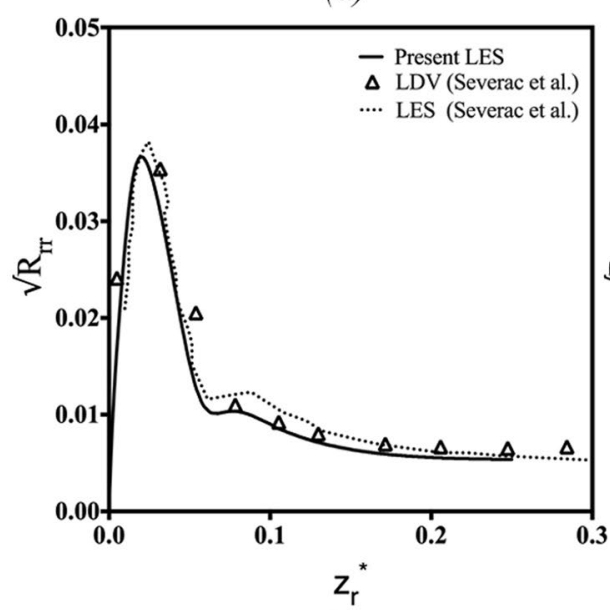

(c)

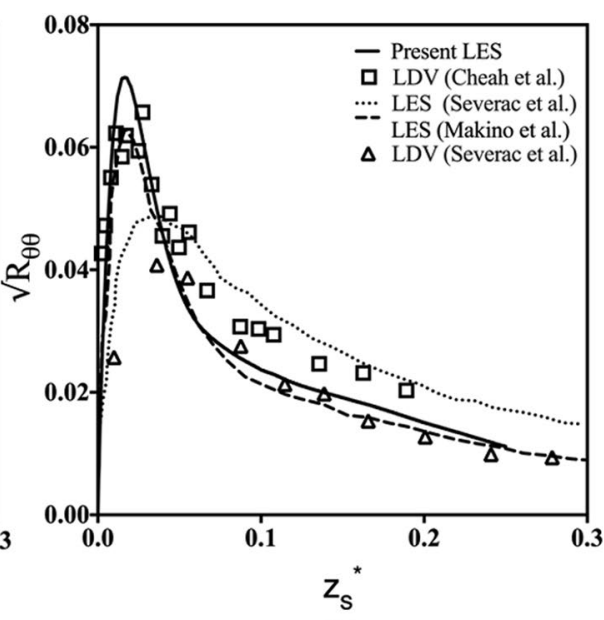

(b)

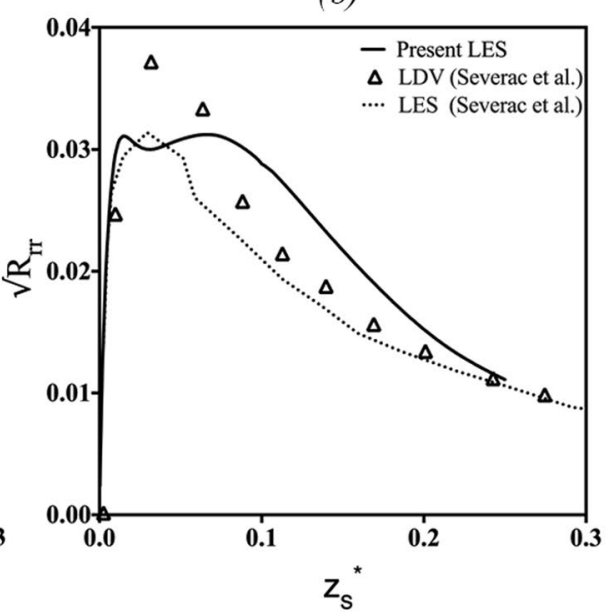

(d)
FIG. 7. The variation of turbulence intensity distributions at $\operatorname{Re}_{\omega}=4 \times 10^{5}$ for smooth cavities: (a) tangential distribution at the rotor, (b) tangential distribution at the stator, (c) radial distribution at the rotor, and (d) radial distribution at the stator. 
Figure 4(d) shows a truncated time series for the normalised tangential fluctuation component $\left(u_{\theta}^{\prime}\right)$ at three different radial positions of $r^{*}=0.25,0.50,0.75$ for $R e_{\omega}=4 \times 10^{5}$. These time series are captured after several dozens of disk rotations, which is sufficient to pass the initial transient state of the simulations. Regardless of the radial location, $u_{\theta}^{\prime}$ tends to show a periodic behavior, but at higher radial locations, fluctuations show high-frequency oscillations than lower radial locations. This shows that the localised model constants of the dynamic Smagorinsky model stabilise the simulations by damping high-frequency modes so that the computation can achieve a longer integration time. Furthermore, our modification of local smoothing on the turbulent model does not produce under or over damping model constants, which might result in the blow-up of the simulations or relaminarisation of the flow field.

Figure 4(e) shows the vector plot of a typical rotor-stator cavity simulation in the $r-z$ plane. The near wall regions of the cavity show three-dimensional behavior for both the rotor and stator. The core region of the cavity separates the rotor and stator boundary layers, and the velocity vectors here are predominately in the tangential direction. The vector plot suggests that the cavity is in regime II or IV ("unmerged regions") in the categorisation of Daily and Nece. ${ }^{4}$

For the presentation of the results, the radial and axial distances are non-dimensionalized with $r^{*}=\frac{r-r_{1}}{r_{2}-r_{1}}$ and $z^{*}=\frac{z}{h}$, respectively. The mean radial and tangential velocity components are both non-dimensionalized on the local rotor speed as $U_{r}^{*}=\frac{U_{r}}{r \omega}$ and $U_{\theta}^{*}=\frac{U_{\theta}}{r \omega}$. All axial plots are extracted at a mid-radial position of the cavity, $r^{*}=0.65$. Here the influence of the finite cavity is minimal.

Figure 5 shows the axial distributions of the mean radial and tangential velocity profiles for $R e_{\omega}=1 \times 10^{5}$ and $4 \times 10^{5}$. Both radial velocity profiles show an inflection point at the rotor layer, are zero in the core region, and become negative in the vicinity of the stator boundary layer where another inflection point is seen. Both tangential velocity profiles achieve their highest value at the rotor surface, decline toward the core region, and become zero at the stator surface. The entrainment coefficient, $K=U_{\theta \text {,core }} / U_{\theta \text {, disk }}$, describes the constant tangential velocity of the core region of the cavity. The current $K$ values at the mid-section of the cavity are $K \approx 0.35$ and 0.37 for $R e_{\omega}=1 \times 10^{5}$ and $4 \times 10^{5}$, respectively. These values agree well with the previous investigation of Séverac et al. ${ }^{10}$ who obtained $K \approx 0.35$ and 0.36 for identical $R e_{\omega}$ and geometric configurations.

Figure 6 shows a comparison of the mean relative tangential velocity, $u_{\theta}=r \omega-U_{\theta}$, in the rotor and stator boundary layers for $R_{\omega}=4 \times 10^{5}$. Here both boundary layers are in a relatively turbulent state compared to the lower $R e_{\omega}$ case. The figure shows the velocities and wall distances normalised by the friction velocity,

$$
\left.u_{\tau}=\left(\left(v \partial U_{\theta}\right) / \partial z\right)_{\mathrm{wall}}^{2}+\left(\left(v \partial U_{r}\right) / \partial z\right)_{\mathrm{wall}}^{2}\right)^{\frac{1}{4}} .
$$

This inner scaling of the velocity distributions allows detailed comparisons of the laminar sublayer, buffer region, and logarithmic layer of the velocity profiles. The laminar sublayer is a linear layer, and the rotor boundary follows strictly this linearity to $z_{r}^{+}=5.0$. It further extends with approximate agreement to $z_{r}^{+} \approx 8.0$ but then the agreement quickly deteriorates. In our simulations, the logarithmic layer starts to emerge at $z_{r}^{+}=10.0$. The thin layer between the laminar sublayer and logarithmic layer $\left(z_{r}^{+} \approx 8.0-10.0\right)$ can be identified as the buffer layer. The stator boundary layer shows the same trend, but the laminar sublayer is found only up to $z_{s}^{+} \approx 5.0$, with the logarithmic layer starting at about $z_{s}^{+}=10.0$. The layer between these two regions defines the buffer layer $z_{s}^{+} \approx 5.0-10.0$, which is broader than those observed in the rotor boundary. A similar trend was observed by Séverac et al. except that they did not observe a buffer region in either boundary layers. The previous experimental results of Cheah et al. ${ }^{40}$ agree well within the laminar sublayer and buffer regions, and the velocity distributions of Makino et al. show excellent agreement throughout the laminar sublayer and buffer region of the boundary layers. To enable the quantitative comparison of our boundary-layer profiles with these experimental and numerical results, all profiles

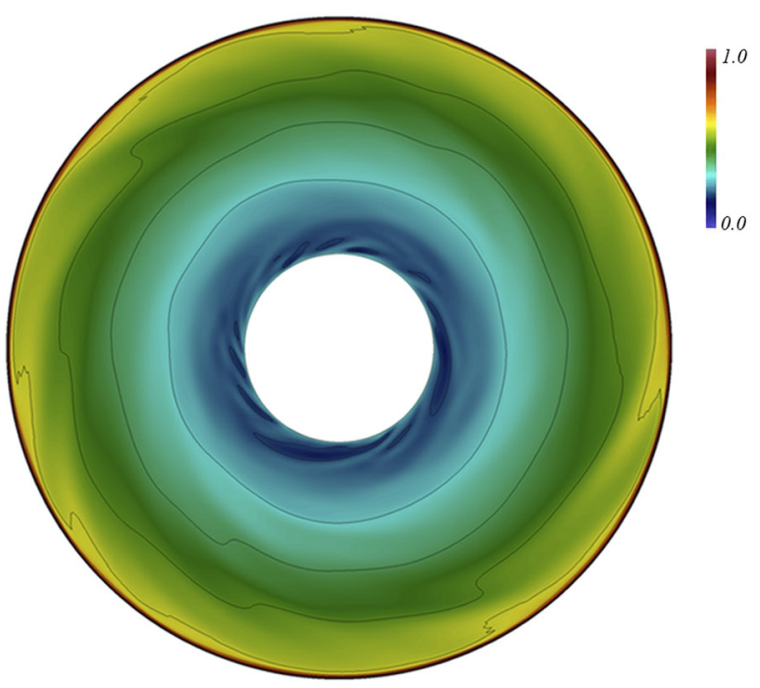

(a)

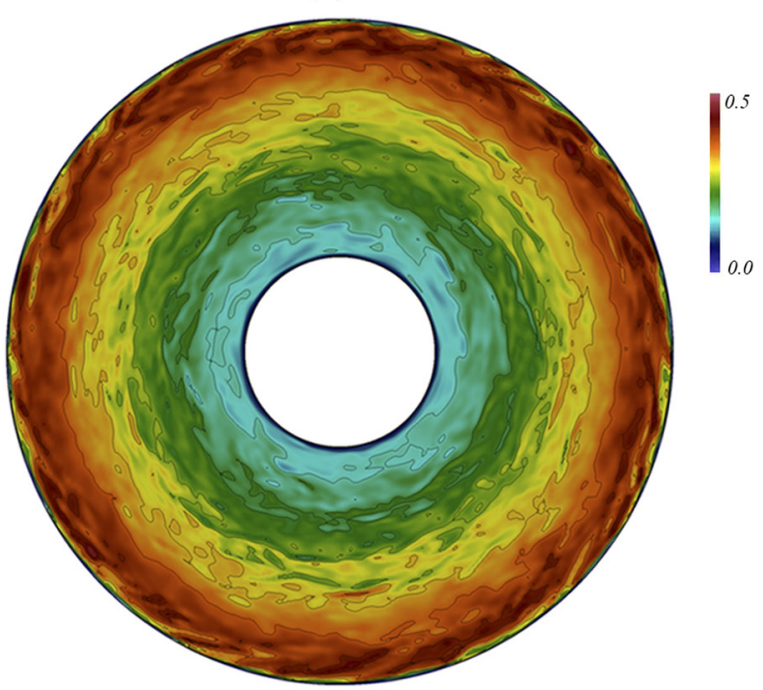

(b)

FIG. 8. Top views of the normalised instantaneous tangential velocity contours for the smooth cavity at $R e_{\omega}=1 \times 10^{5}$ : (a) near the rotor boundary $\left(z^{*}=0.025\right)$ and (b) near the stator boundary $\left(z^{*}=0.95\right)$. 
are fitted to the well-known logarithmic velocity profile (see Ref. 41),

$$
u_{\theta}^{+}=\frac{1}{\kappa} \log \left(z^{+}\right)+B .
$$

Here $\kappa$ is the von Kármán constant and $B$ is the intersection, and the reference values of $\kappa \approx 0.4$ and $B=5.5$ can be defined for a smooth-wall circular turbulent pipe flow. ${ }^{42}$ Table III shows a summary of configurations and a comparison of the gradient $\kappa^{-1}$ and intersection values of the present LES with previous investigations when their respective velocity profiles are fitted to Eq. (8). The fitted values are within an acceptable range of both previous numerical and experimental results. However, the peak values of the velocity profiles do not agree well with either of the experimental investigations, which indeed do not agree with each other. We believe that this is due to subtly different configurations and operational
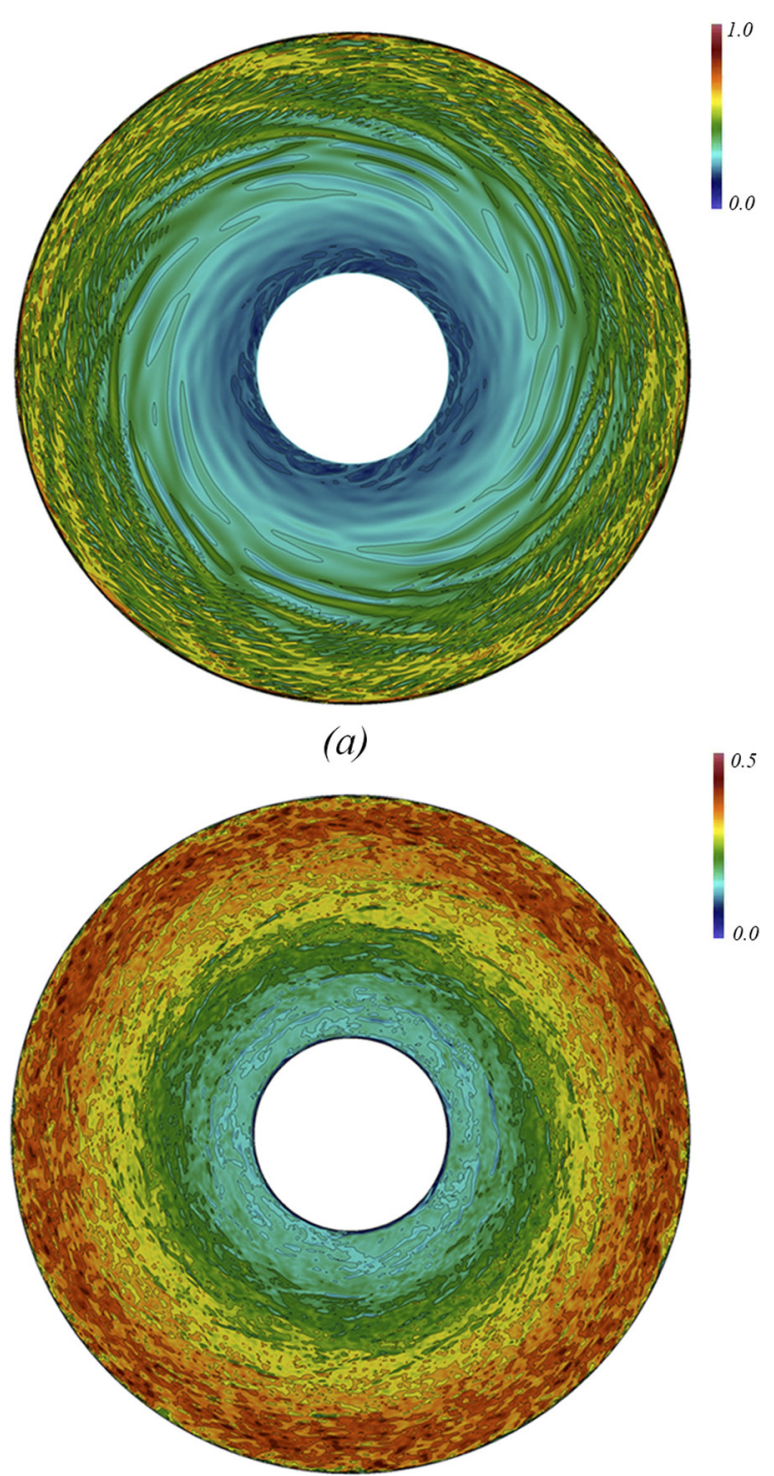

(b)

FIG. 9. Top views of the normalised instantaneous tangential velocity contours for the smooth cavity at $\operatorname{Re}_{\omega}=4 \times 10^{5}$ : (a) near the rotor boundary $\left(z^{*}=0.025\right)$ and $(b)$ near the stator boundary $\left(z^{*}=0.95\right)$. conditions between the two experiments and as compared to our system.

Figure 7 shows the radial and tangential turbulence intensity components normalised using $\sqrt{R_{\theta \theta}}=\sqrt{\frac{\overline{u_{\theta}^{\prime} u_{\theta}^{\prime}}}{(r \omega)^{2}}}$ and $\sqrt{R_{r r}}=\sqrt{\frac{\overline{u_{r}^{\prime} u_{r}^{\prime}}}{(r \omega)^{2}}}$. Note that axial distances are measured from their respective boundary surfaces; e.g., $z_{s}^{*}=0$ is the stator surface for the stator velocity plot, and vice versa. The axial locations of the peaks of the tangential turbulence intensity of the rotor and stator distributions are at $z_{r}^{*}=0.013$ and $z_{s}^{*}=0.018$, respectively. Despite well-agreed peak locations, the current LES overestimates the peak values by $13 \%$ in the rotor boundary and $14 \%$ in the stator boundary, as compared to

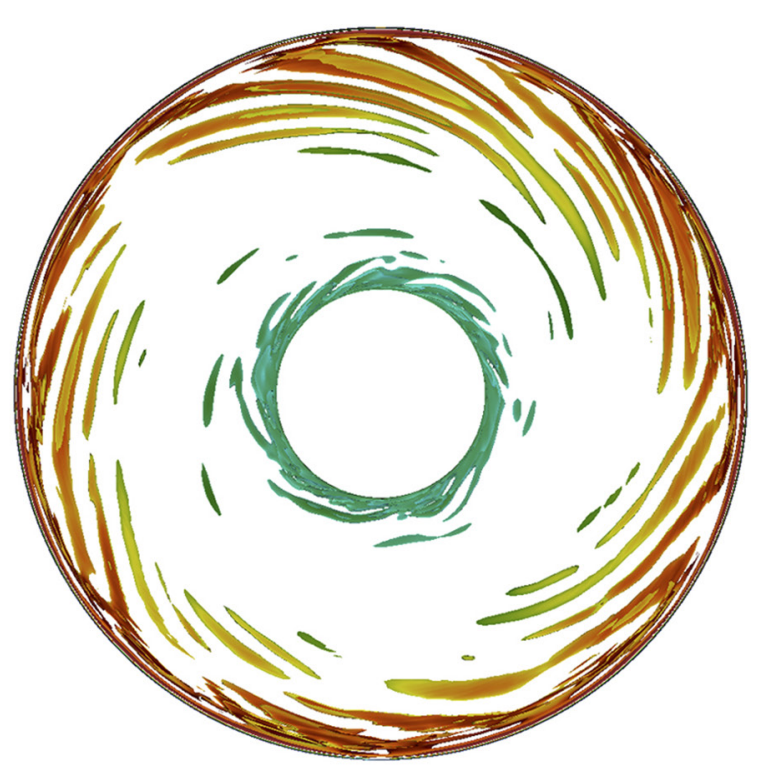

(a)

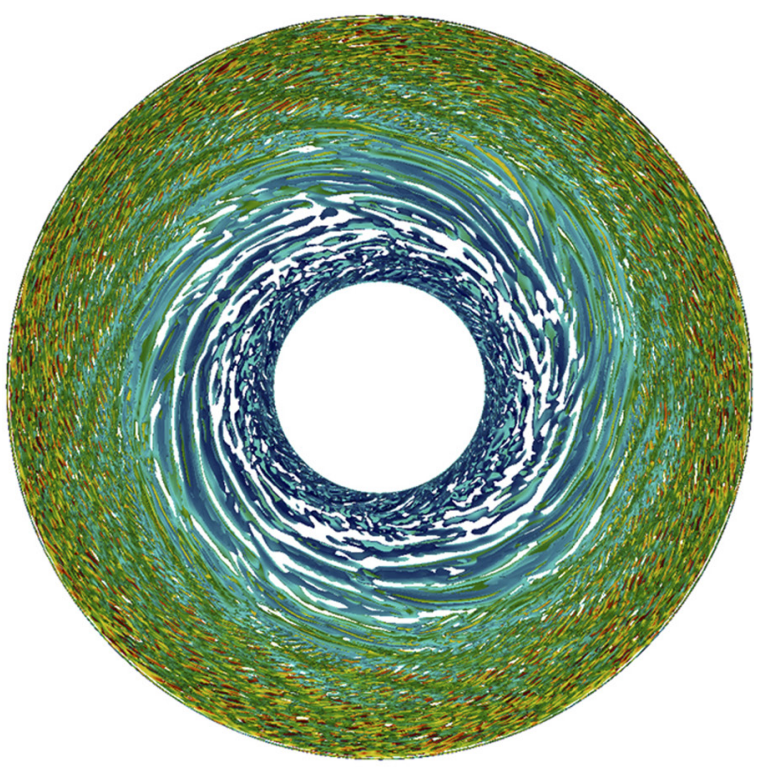

(b)

FIG. 10. Top view of iso-surfaces of $\lambda_{2}$ vortex structures near the rotor boundary layer at (a) $R e_{\omega}=1 \times 10^{5}$ and (b) $R e_{\omega}=4 \times 10^{5}$ for smooth cavities. 
the same investigation of Makino et al. ${ }^{11}$ Similarly, the peaks of the radial intensity distributions are located inside the boundary layers at $z_{r}^{*}=0.019$ and $z_{s}^{*}=0.017$ for the rotor and stator boundary layers, respectively.

Figures 8 and 9 show instantaneous tangential velocity contours near the rotor $\left(z^{*}=0.025\right)$ and stator $\left(z^{*}=0.95\right)$ boundary layers; all velocity contours are normalised against $U_{\theta, \max }=r_{2} \omega$. The figures show that the higher Reynolds number leads to turbulent behavior within both boundary layers. Regardless of the Reynolds number, both boundary layers show intense behavior near the rotor hub, suggesting that the rotor hub provides destabilising effects on both the rotor and stator. It is known that the stator boundary layers become turbulent at much lower Reynolds numbers than the rotor boundary layers, ${ }^{10}$ and here contours near the stator boundary layers show much higher turbulent activity. Even at $R e_{\omega}=4 \times 10^{5}$, the inner radial region of the rotor is not yet fully turbulent, but there are axisymmetric elongated structures at larger radial positions. These are indicative of a turbulent flow in the rotor boundary layer.

Figure 10 gives an iso-surface representation of vortical structures in the rotor boundary layer. The $\lambda_{2}$ criterion, ${ }^{44} \mathrm{a}$ well-known vortex identification method, has been used to extract these vortex structures. Note that the colour scheme in the figure does not correspond to the strength of the vortex cores, but represents the magnitude of the local tangential velocity. At $R e_{\omega}=1 \times 10^{5}$, some spiral patterns start to emerge in the boundary layer, but there are no fine scale structures toward the peripheral of the disk. At $R e_{\omega}=4 \times 10^{5}$, the boundary layer is populated with elongated spiral structures at the low $R e_{\omega, \text { local }}$ regions of the cavity and, as the local Reynolds number increases, these structures turn into an array of spiral arms around the rotor disk. These spiral patterns further breakdown into fine-scale turbulent structures at high $R e_{\omega, \text { local }}$ locations, indicative of a fully turbulent region. We find approximately 14-15 spiral arms around the rotor boundary layer, with an angle of inclination $\epsilon \approx-18^{\circ}$ to the tangential direction. These observations are in close agreement with previous investigations. ${ }^{10,11}$

The velocity contours and iso-surfaces of the rotor boundary do not show any Type I spiral patterns (as would be identified by positive $\epsilon$ ) which suggests that turbulent transition here is not due to an inviscid cross-flow instability. This is consistent with the findings of Makino et al. ${ }^{11}$ who suggested that the structures are a result of the Type II streamline-curvature instabilities first identified by Itoh. ${ }^{6}$ In particular, Makino et al. suggested that the turbulent transition of the rotor-stator cavity could be a result of interactions between the dominant Type II modes and their secondary instability patterns, which is denoted as "mechanism $B$ " in the investigation of Faller. ${ }^{45}$

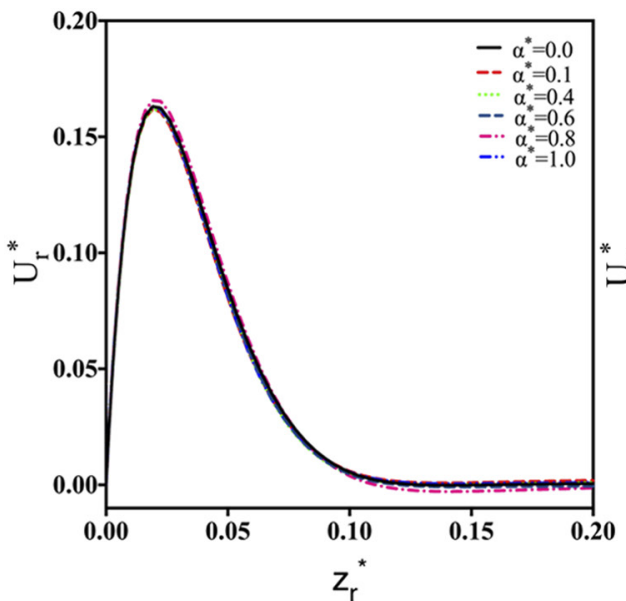

(a)

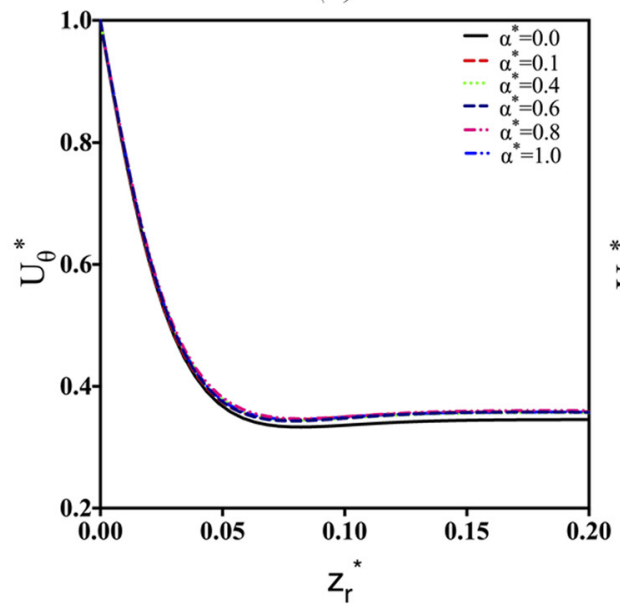

(c)

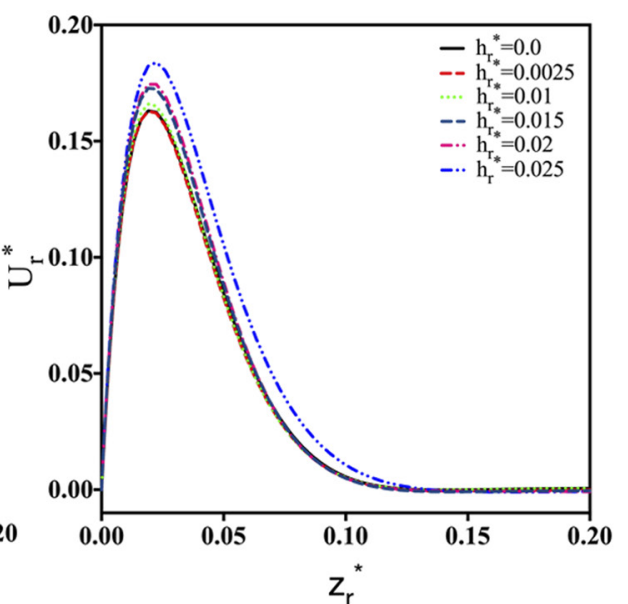

(b)

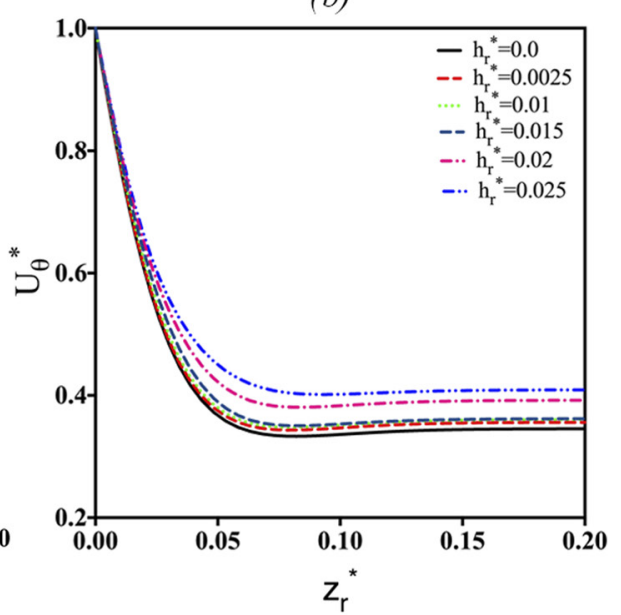

(d)
FIG. 11. A comparison of mean velocity profiles at the rotor boundary layer for $R e_{\omega}=1 \times 10^{5}$ for different model parameters for rough wall cavities: $\alpha^{*}=0.1-1.0$ and $h_{r}^{*}=0.005$ for (a) radial and (c) tangential profiles and $h_{r}^{*}=0.0025-0.025$ and $\alpha^{*}=0.2$ for (b) radial and (d) tangential profiles. 

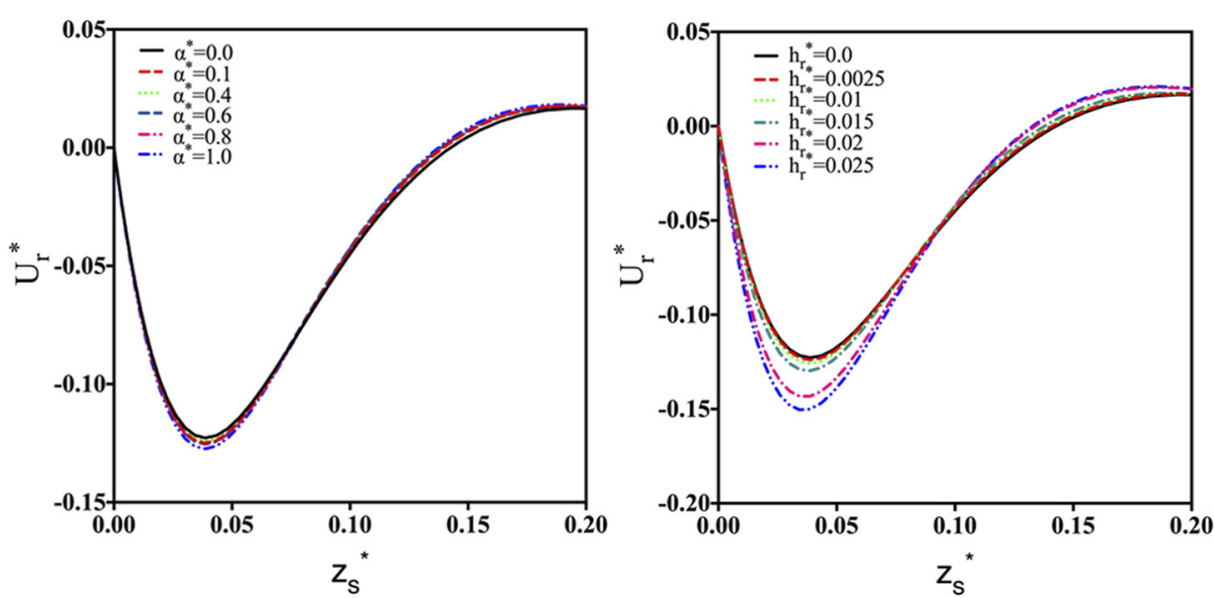

(a)

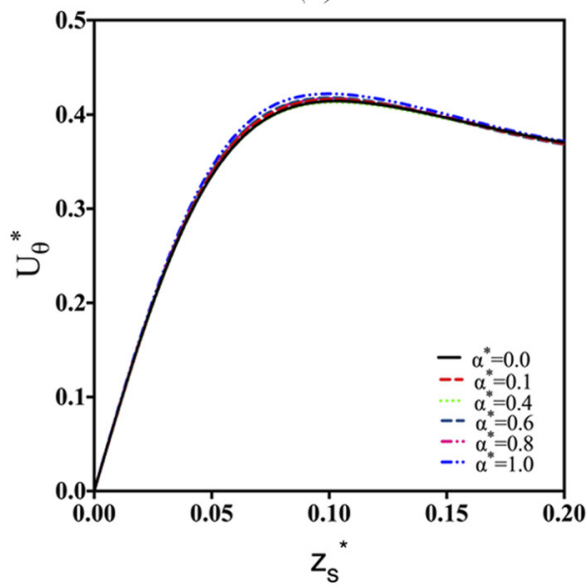

(c)

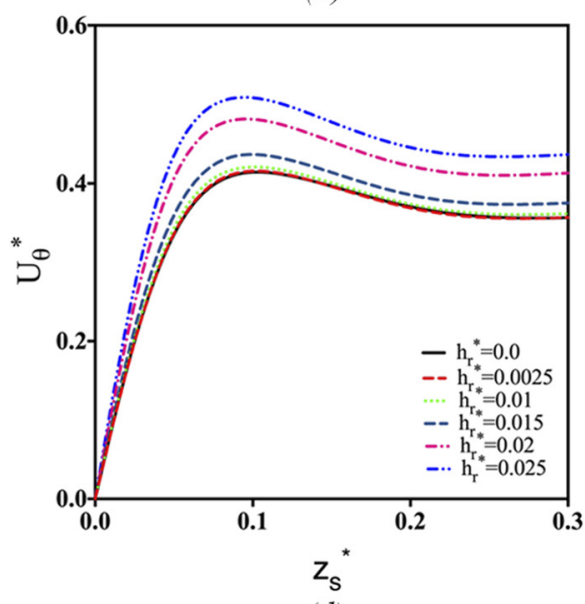

(d)
FIG. 12. A comparison of mean velocity profiles at the stator boundary layer for $R e_{\omega}=1 \times 10^{5}$ for different model parameters for rough wall cavities: $\alpha^{*}=0.1-1.0$ and $h_{r}^{*}=0.005$ for (a) radial and (c) tangential profiles and $h_{r}^{*}=0.0025-0.025$ and $\alpha^{*}=0.2$ for (b) radial and (d) tangential profiles.

\section{B. Rough wall rotor-stator cavity}

This section presents test cases of $R e_{\omega}=1 \times 10^{5}$ and $4 \times 10^{5}$ for rotor-stator cavities with rough rotor and smooth stator walls. Initially the response of the mean velocity distributions to different model constants $h_{r}^{*}$ and $\alpha^{*}$ is presented. Subsequently, the turbulent flow properties and flow visualisations of the rough rotor and smooth stator boundary layers are evaluated and compared with our smooth rotor-stator cavity results from Sec. III A. It is important to remember that the model constants $h_{r}^{*}$ and $\alpha^{*}$ are not calibrated against physical roughness conditions and are merely empirical constants within our simulations. However, these model constants do enable the qualitative study of the relative effects of a rough rotor wall by observing the shifts in the flow-field distributions relative to our smooth rotor-stator simulations. In the future, our model constants could be replaced with experimental or DNS calibrated values to represent real-world rough surfaces.

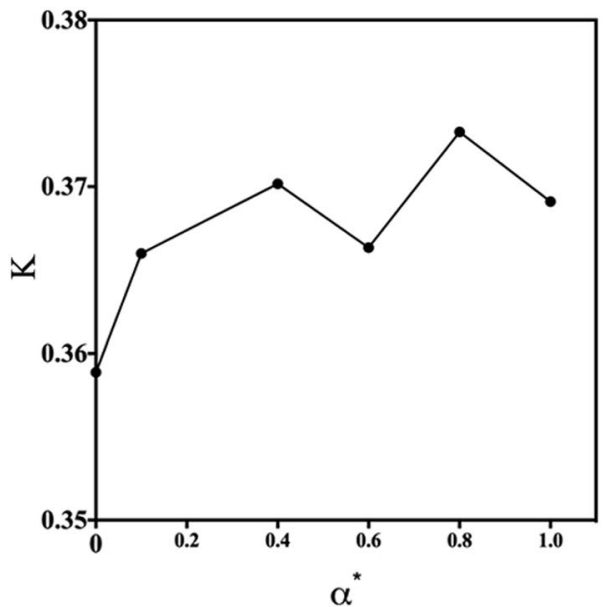

(a)

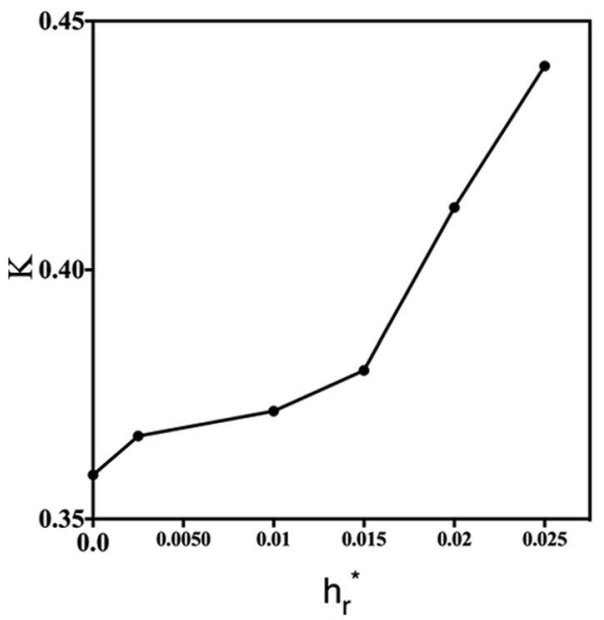

(b)
FIG. 13. The variation of $K$ with model constants for rough wall cavities: (a) $\alpha^{*}$ and (b) $h_{r}^{*}$, at $R e_{\omega}=1 \times 10^{5}$. 


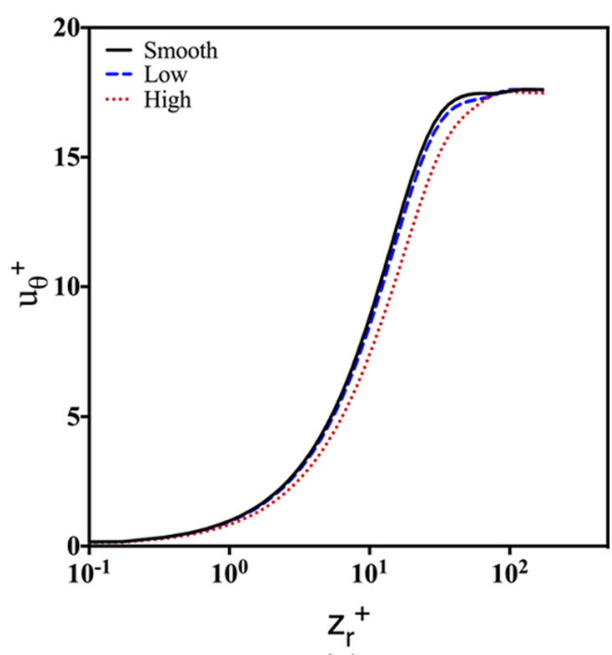

(a)

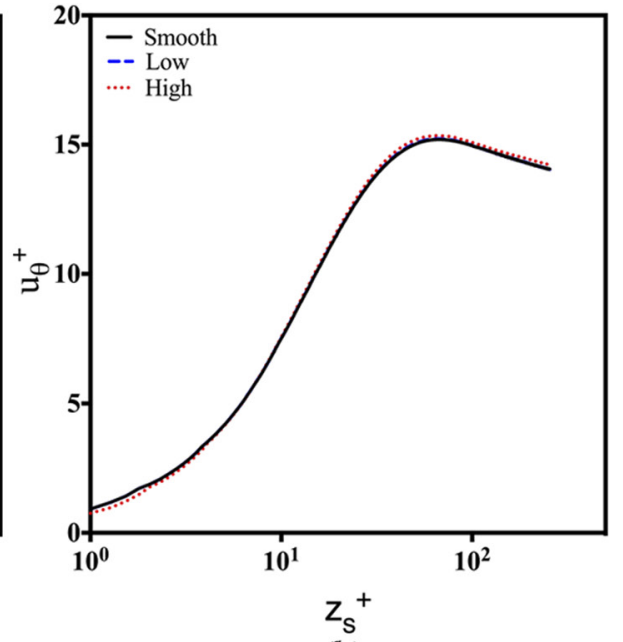

(b)
FIG. 14. Influence of low and high rotor boundary roughness on mean tangential velocity profiles at $R e_{\omega}=4$ $\times 10^{5}$ for rough wall cavities: (a) rotor boundary layer and (b) stator boundary layer.
Figure 11 shows the variations of tangential and radial velocity profiles of the rotor boundary layer under different model constant values. In particular, we consider $\alpha^{*}=0.1-1.0$ at constant $h_{r}^{*}=0.005$ and $h_{r}^{*}=0.0025-0.025$ at constant $\alpha^{*}=0.2$. By observing these velocity plots, it is clear that the height parameter $h_{r}^{*}$ has a significant effect on the mean velocity profiles. Increasing the height parameter increases the peak value of the radial profiles, and the profiles show an outward shift compared to the smooth velocity profile. However, increasing $\alpha^{*}$ tends to shift the velocity profiles inward and leads to a marginal decrease in their peak values (except for the highest $\alpha^{*}$ values). The tangential velocity profiles tend to shift in the outward direction and, as might be expected, increasing the height parameter also has a significant impact on the tangential velocity profiles. The simulations with numerical roughness layers higher than the model constants $\alpha^{*}=0.4$

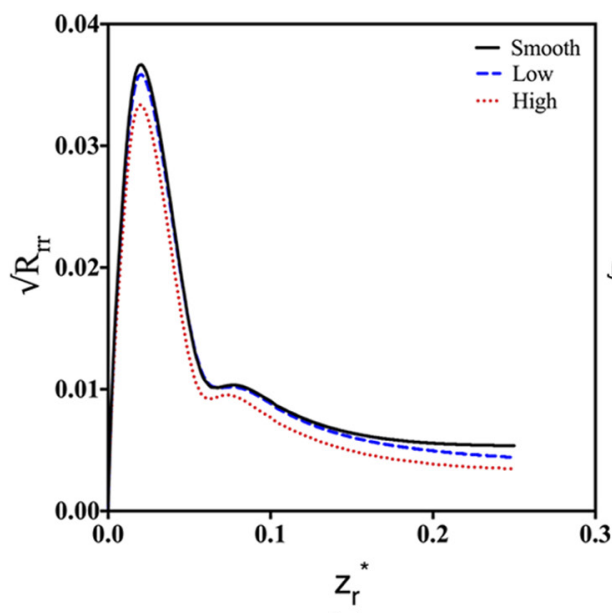

(a)

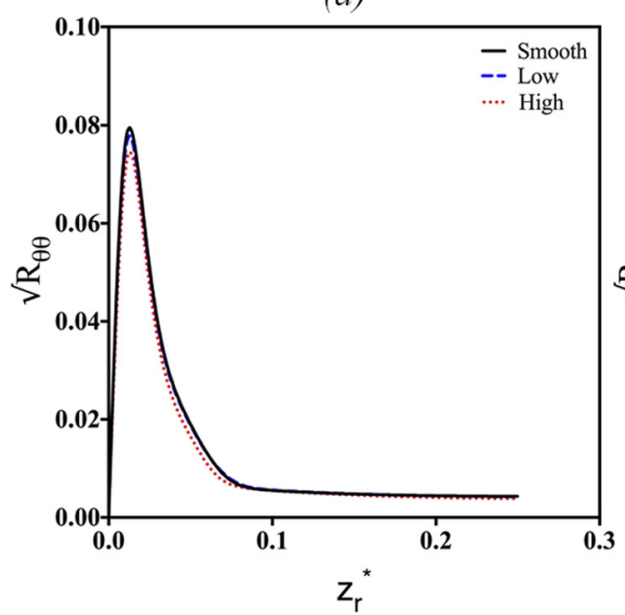

(c)

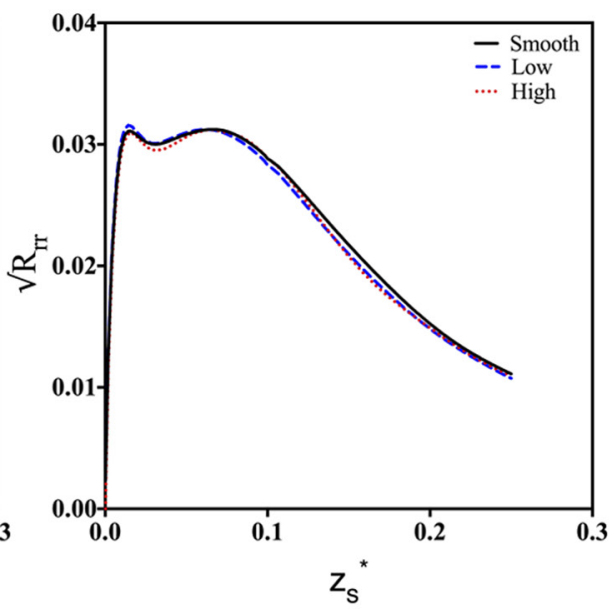

(b)

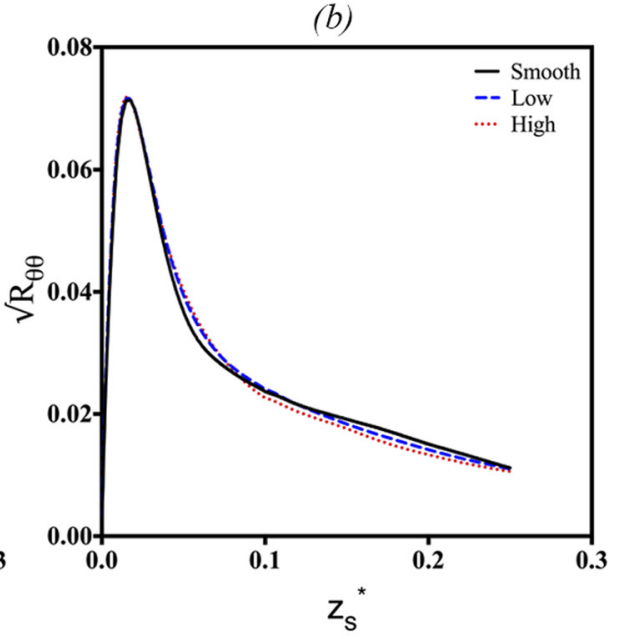

(d)
FIG. 15. Influence of rotor boundary roughness on turbulence intensity profiles at $R e_{\omega}=4 \times 10^{5}$ for rough wall cavities: (a) radial intensity profile at the rotor boundary, (b) tangential intensity profile at the stator boundary, (c) radial intensity profile at the rotor boundary and (d) tangential intensity profile at the stator boundary. 


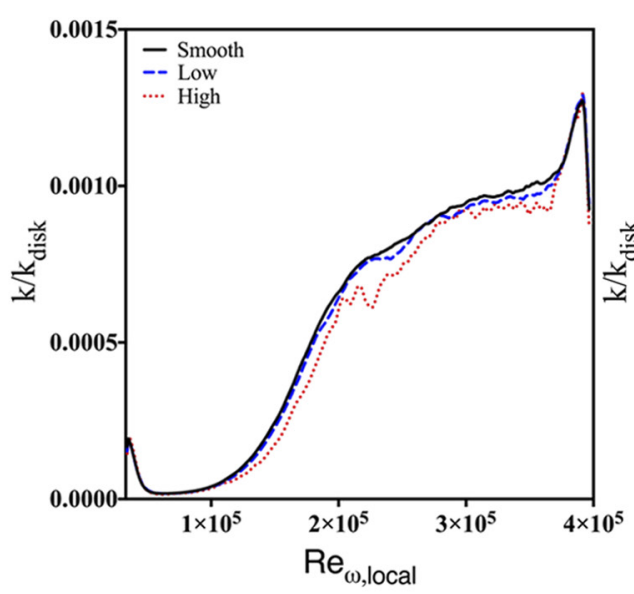

(a)

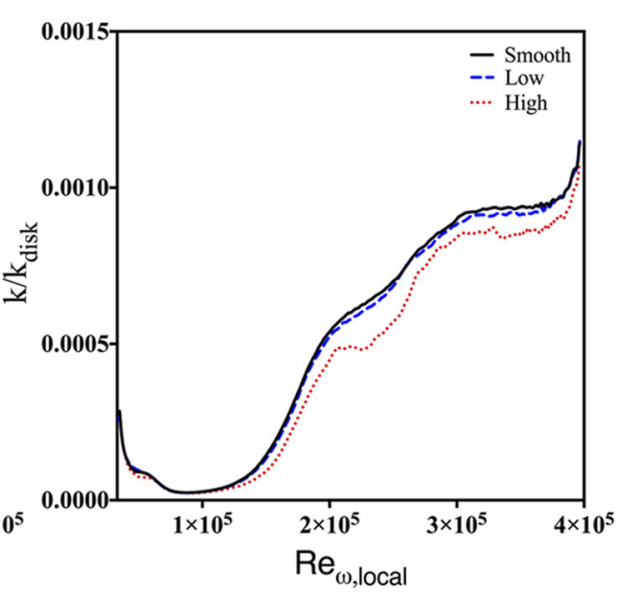

(b)
FIG. 16. Influence of low and high rotor boundary roughness on turbulent kinetic energy distributions in the radial direction for rough wall cavities in $R e_{\omega}=4 \times 10^{5}$ : (a) at $z^{*}=0.0125$ and (b) at $z^{*}=0.04$. and $h_{r}^{*}=0.005$ have significant effects on the smooth stator layer of the cavity. Figure 12 shows the corresponding tangential and radial velocity distributions of the stator boundary layer for the same model constant ranges shown in Fig. 11. The velocity profiles suggest that intense roughness causes an increase in the near-wall peak values. Under the higher roughness values, the peaks are slightly relocated toward the stator wall, but this is more pronounced in the simulations with higher $h_{r}^{*}$ values.

The recent investigation of Özkan et al. ${ }^{17}$ imposed roughness effects through a RANS rough wall function, and their tangential velocity profiles show the same outward shifting trend when the boundary layers are susceptible to increased roughness effects. However, their radial velocity profiles tend to shift inward and with diminished peak values. Alveroglu et al. ${ }^{16}$ imposed roughness effects using a partial slip approach within the BEK boundary layer. In this approach, radial velocity profiles of azimuthally anisotropic roughness (radial grooves) in the Ekman layer show the same tendency as the radial profiles of Fig. 11. The disagreement with the radial profiles of Özkan et al. could be due to the incompatibility of the type of the roughness. The combined effects of $h_{r}^{*}, \alpha_{i}^{*}=\left(\alpha_{r}^{*}, \alpha_{\theta}^{*}, \alpha_{z}^{*}\right)$, and the shape function are important factors to uniquely determine the type of the roughness, and the different combinations provide a mechanism to simulate many different roughness types. This sensitivity to the precise form of the roughness was also demonstrated by Garrett et al. ${ }^{15}$

Figure 13 shows the variation in the entrainment coefficient under different $\alpha^{*}$ and $h_{r}^{*}$ values. An increase in either $\alpha^{*}$ or $h_{r}^{*}$ shows an increase in $K$ from $K \approx 0.35$ for smooth boundary layers.

Turbulent rotor boundary layers are achieved at $R e_{\omega}=4 \times 10^{5}$, and two test cases low $\left(h_{r}^{*}=0.00125\right)$ and high $\left(h_{r}^{*}=0.00375\right)$ have been simulated here for $\alpha^{*}=0.4$. The parameters are kept at moderate values, in comparison to the previous test cases, so as to achieve an outer-layer similarity. ${ }^{38}$ Figure 14 shows mean turbulent velocity profiles represented in the form of Eq. (8). The rotor boundary layer shows the effect of roughness via velocity deflection effects, as also demonstrated in previous investigations including those of Busse and Sandham. ${ }^{18}$ However, the smooth stator boundary layer does not show significant changes due to the low values of $\alpha^{*}$ and $h_{r}^{*}$ used over the rotor.

Figure 15 shows the tangential and radial turbulent intensities in the rotor and stator boundary layers. The distribution evident in the stator boundary confirms that the roughness effects on the rotor side are confined within the inner region of the rotor boundary. Increased roughness effects result in a decrease in the near-wall peak of the turbulence intensity profiles at the rotor boundary, and this may be due to the damping nature of the roughness term in the momentum equation. A similar trend was observed in the previous experimental investigations of Krogstad et $a .^{46}$ and the DNS investigations of Orlandi et al., ${ }^{47}$ Flores and Jiminez, ${ }^{33}$ and Busse and Sandham. ${ }^{18}$ Busse and Sandham reported on the possibility of peak relocation near the wall surfaces, but peaks of our current distributions are located at nearly the same axial location. The damping effects of the tangential turbulent intensities are less intense compared to the equivalent radial turbulence
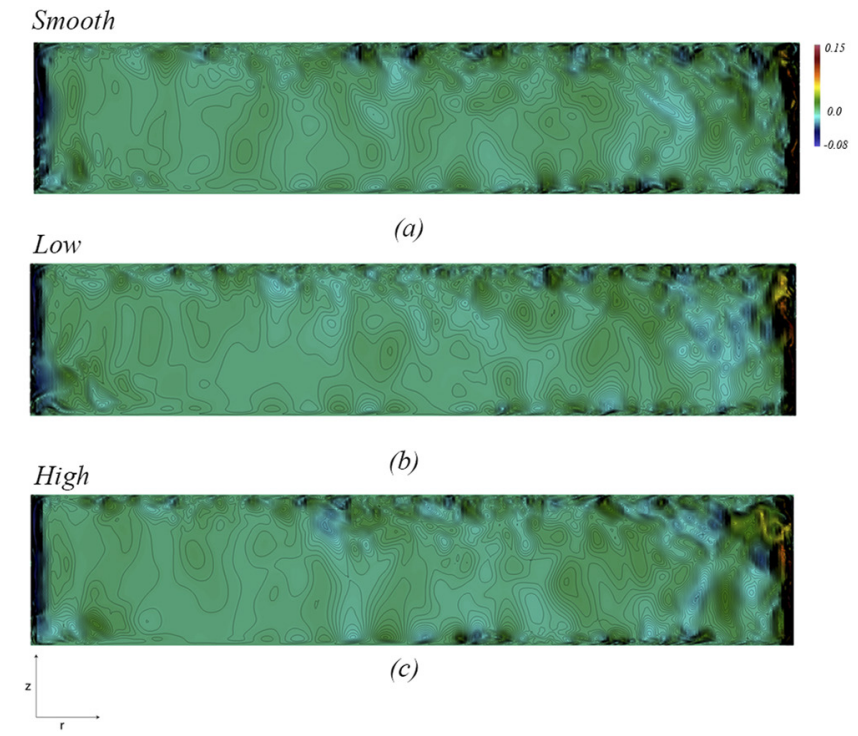

FIG. 17. A comparison between the normalised instantaneous axial velocity contours across the $r-z$ plane of the entire smooth and rough wall cavities for $R e_{\omega}=4 \times 10^{5}$ : (a) smooth, (b) low, and (c) high. 


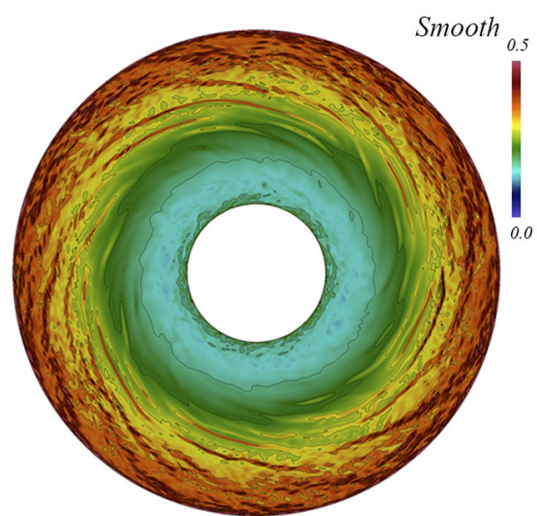

(a)

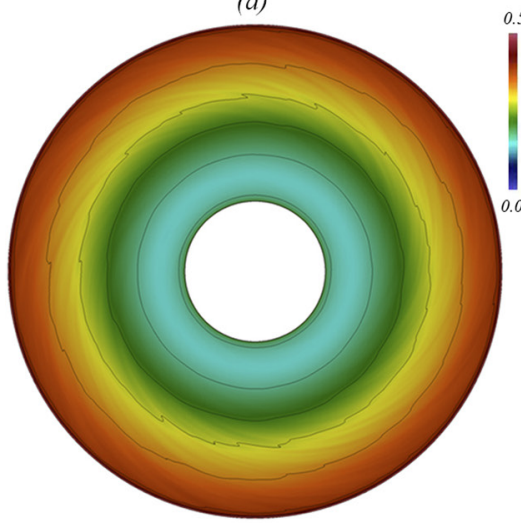

(d)

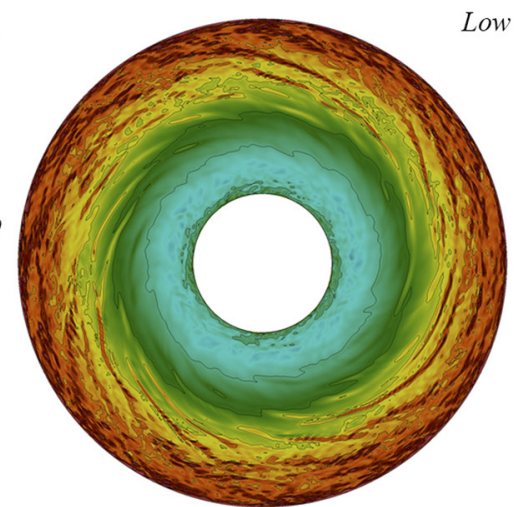

(b)

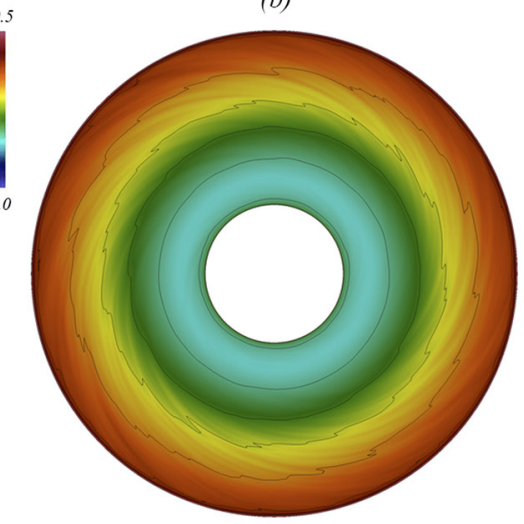

(e)

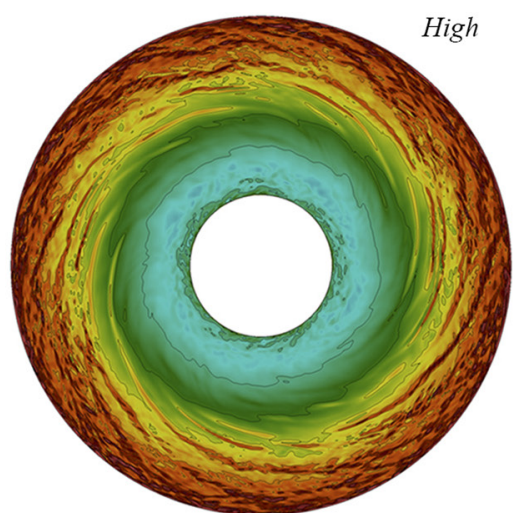

(c)

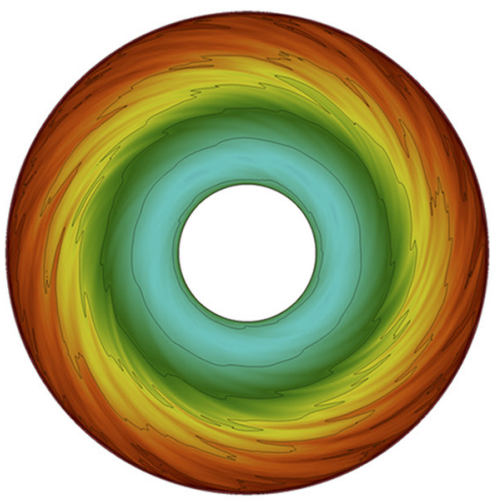

(f)

FIG. 18. A comparison between the normalised tangential velocity contours near the rotor boundary layer $\left(z^{*}=0.04\right)$ for smooth and rough wall cavities at $R e_{\omega}=4 \times 10^{5}$. Instantaneous velocity contours: (a) smooth, (b) low, and (c) high and mean velocity contours: (d) smooth, (e) low, and (f) high.

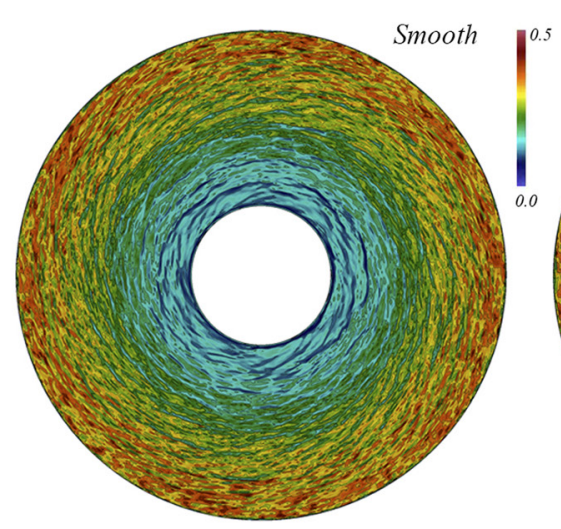

(a)

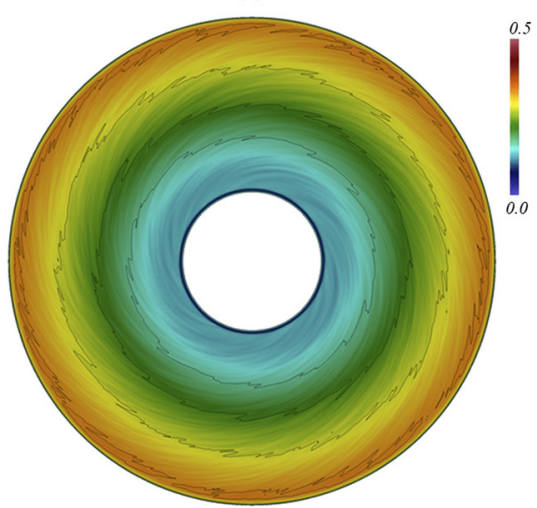

(d)

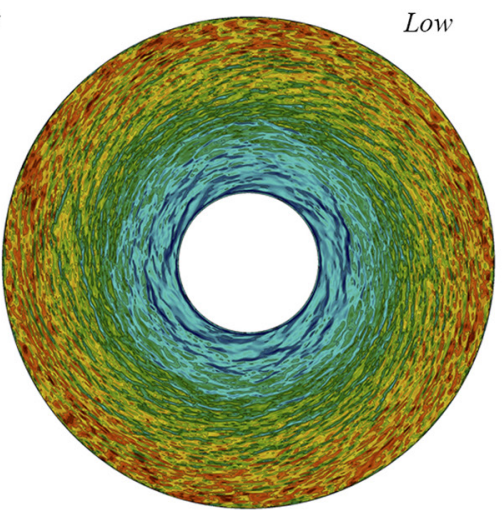

(b)

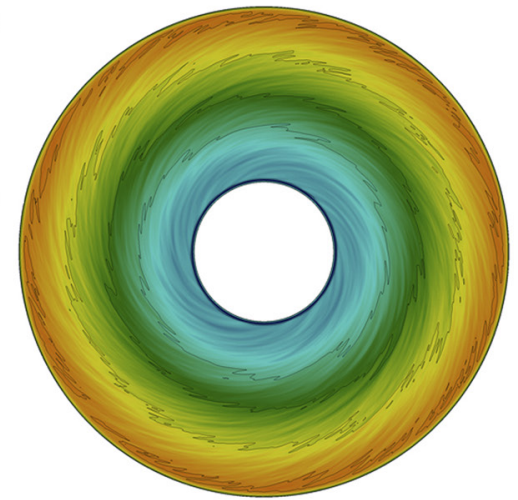

(e)

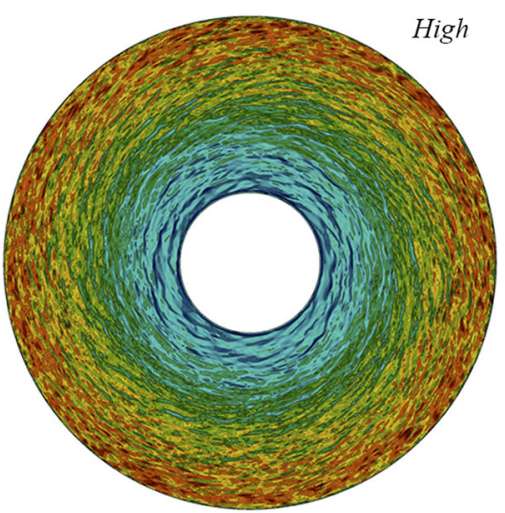

(c)

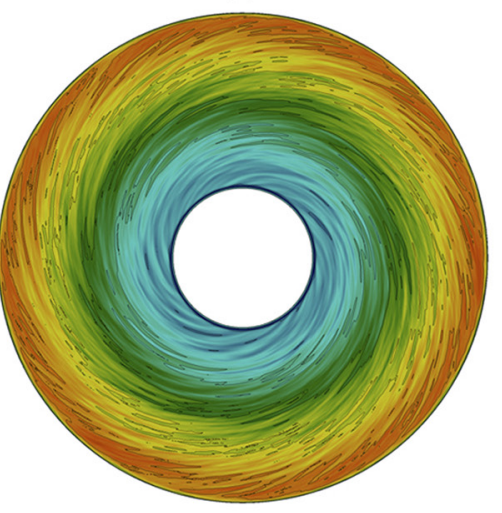

(f)

FIG. 19. A comparison between the normalised tangential velocity contours near the stator boundary layer $\left(z^{*}=0.975\right)$ for smooth and rough wall cavities at $R e_{\omega}=4 \times 10^{5}$. Instantaneous velocity contours: (a) smooth, (b) low, and (c) high and mean velocity contours: (d) smooth, (e) low, and (f) high. 
intensity distributions, and the tangential distribution of the rotor side collapses at a much shorter axial distance than the radial turbulence intensity distributions.

The mean and turbulent distributions are extracted from the mid-section of the cavity and are less likely to reflect the local Reynolds number dependency on the finite nature of the rotor-stator cavities. The turbulent kinetic energy $k$ is another important parameter, which gives a quantitative understanding of the mean kinetic energy associated with eddies in the turbulent flow. For the sake of comparison, radial distributions of the turbulent kinetic energy plots are constructed at two axial distances, $z^{*}=0.0125$ and 0.04 , from the rotor surface. These distances are inside and just outside of the numerical roughness layer, enabling us to investigate the behavior of turbulent kinetic energy both inside and outside the numerical roughness layer.

Figure 16 illustrates the kinetic energy distribution along the radial direction, normalized by $k_{d i s k}=0.5\left(U_{\theta, \max }\right)^{2}$. There is a clear reduction in the kinetic energy distributions in the axial direction, and the effects of the rotor hub are more apparent at smaller radial locations. In both cases, the magnitude of the kinetic energy is fairly constant up to $R e_{\omega, \text { local }}=1.1 \times 10^{5}$, and after this, there is a gradual increase in kinetic energy. The damping effects at the rough walls are also apparent in these kinetic energy plots. As the local Reynolds number increases, the disparity between the distributions increases-this suggests that the roughness effects are more pronounced at higher Reynolds numbers. However, at about $R e_{\omega, \text { local }} \approx 2.6 \times 10^{5}$ $-3.0 \times 10^{5}$, there is a sudden increase in kinetic energy in the rough wall cases.

Figure 17 shows the axial velocity contours of rough and smooth wall cases at the $r-z$ plane. In these cases, there are no significant differences between the magnitudes of the axial velocity components. However, the inner regions of the stator boundary layers of rough wall cavities are distributed with more vortex structures compared to the smooth wall cavity counterpart. In the rough wall cases, the structures in the core region and the rotor boundary layers are vertically elongated, and this is more apparent in the structures at the mid-radial positions. Another difference is that the upper junction between the stator layer and outer wall is more disturbed in rough wall cavities, which signals a plausible path for the transportation of roughness effects to downstream regions (or to the stator) even at these low roughness parameters.

Figure 18 illustrates the instantaneous and mean tangential velocity contours close to the rotor boundary layer, extracted at $z^{*}=0.04$. The instantaneous velocity contours of rough wall cavities show more disturbed structures at higher Reynolds number regions. Here the size of the structures gradually increases with increased roughness effects. The mid and low $R e_{\omega, \text { local }}$ regions show the same tendency, but the effects are less significant. The mean velocity contours show the similar growth in structures at higher Reynolds numbers, but velocity contours at mid and $R e_{\omega, \text { local }}$ regions are almost similar. This suggests that the structures in higher Reynolds number regions are more persistent compared to the structures at mid and low Reynolds number regions.

Figure 19 shows the resulting instantaneous and mean tangential velocity contours on the stator boundary layer at $z^{*}=0.975$. The instantaneous tangential velocity contours on the stator show the same trend that was observed in the rotor boundary layer: At higher Reynolds number regions, the roughness effects become more intense as the roughness height increases in the rotor boundary. These effects are also reflected on the mean velocity contours, but, unlike in the rotor boundary layer, the contours suggest that the disturbances spread into the inner regions of the cavity. This is of no surprise as the stator boundary layers promote radial flow into the cavity because of the favourable pressure gradients in the inward radial direction. This visual evidence suggests that the rotorstator cavities can be disturbed even for small roughnesses, and disturbance patterns at large radial positions of the cavity could be the result of a disturbance propagation mechanism. This is explained in Fig. 17.

Figure 20 shows the $\lambda_{2}$ iso-surface representation of some arbitrary vortex structures at $z^{*}=0.04$. These structures are captured at identical locations for both smooth and rough walls. By a direct comparison of both cases, it is clear that some structures at the outer edge of the rough rotating wall are plumper than their smooth-wall counterparts. A similar observation was made in the investigation of Busse and Sandham. ${ }^{18}$ Moreover, as shown in Fig. 21, a similar trend can been observed in the stator boundary.

The rough wall simulations do not show a significant difference in the turbulent transition properties of the rotor
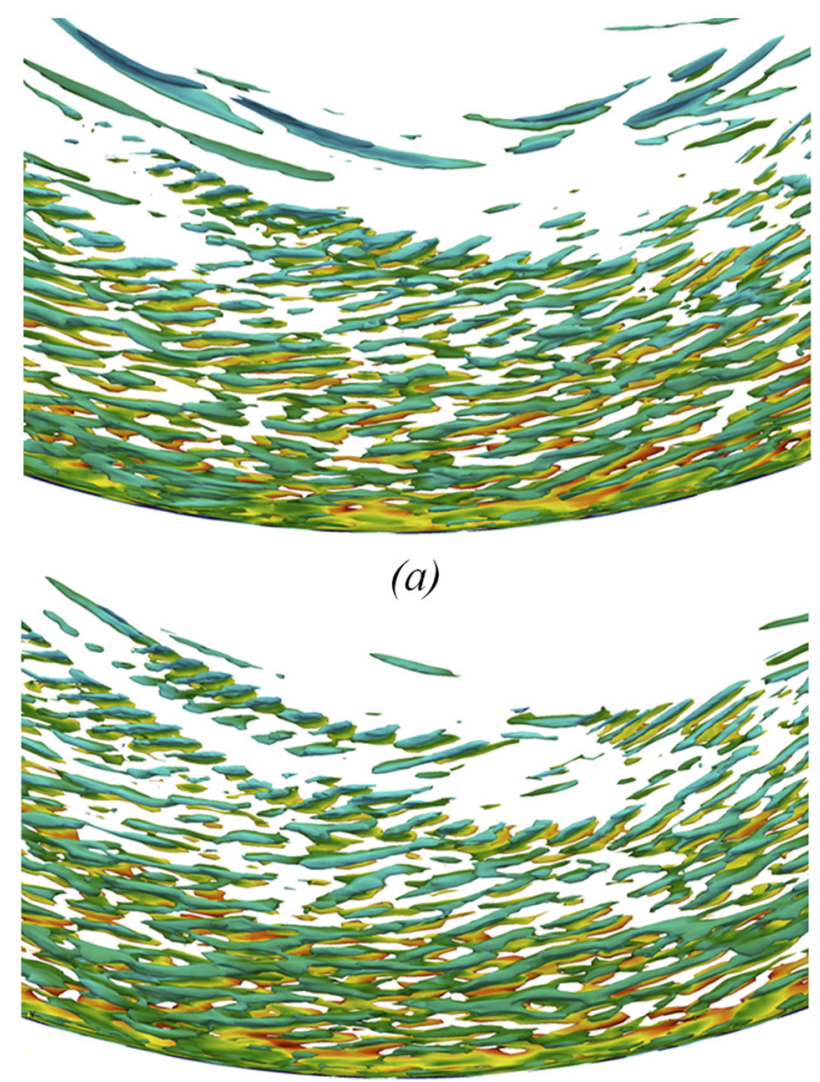

(b)

FIG. 20. A comparison between the $\lambda_{2}$ iso-surfaces of vortex structures near the rotor boundary layer for smooth and rough wall cavities at $R e_{\omega}=4 \times 10^{5}$ $\left(z^{*}=0.04\right)$ : (a) smooth and (b) high. 


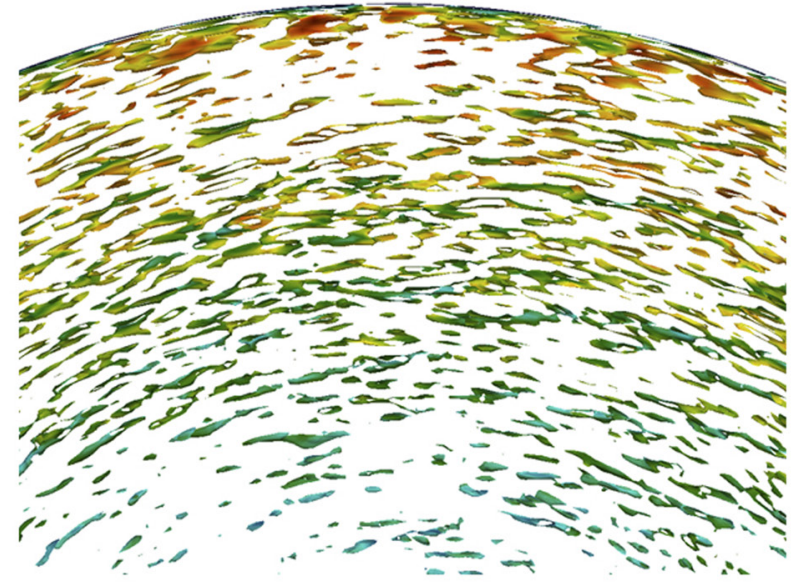

(a)

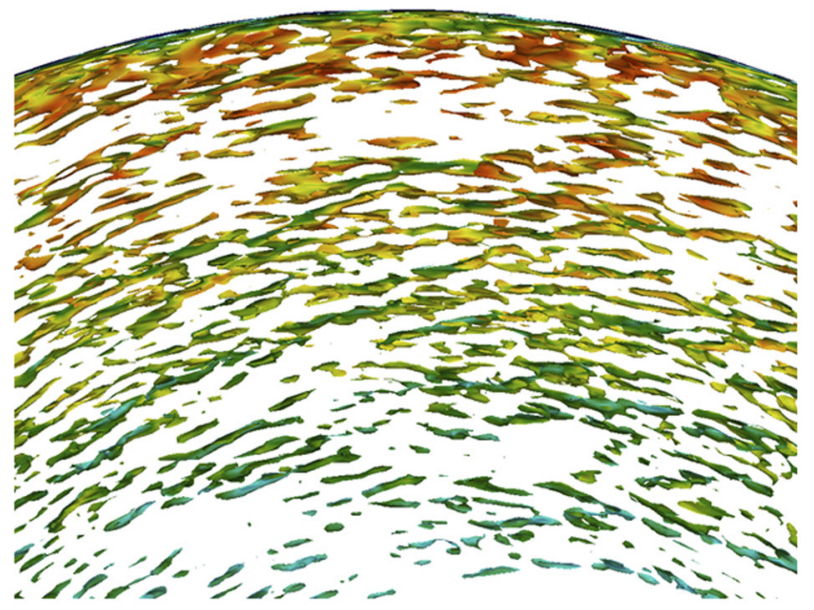

(b)

FIG. 21. A comparison between the $\lambda_{2}$ iso-surfaces of vortex structures near the stator boundary layer for smooth and rough wall cavities at $R e_{\omega}=4 \times 10^{5}$ $\left(z^{*}=0.975\right)$ : (a) smooth and (b) high.

boundary layers. This could be due to the small values of $\alpha^{*}$ and $h_{r}^{*}$ used in our current simulations. Previous investigations $^{12,13}$ of the rotating disk boundary layer have also found only subtle changes in the transition Reynolds number, $R e_{c}$, for small relative roughness levels.

\section{DISCUSSION AND CONCLUSIONS}

The first part of our investigation served to validate flows within smooth rotor-stator cavities using a general purpose CFD library, OpenFOAM. Simulations were conducted in two widely used operational Reynolds numbers, $R e_{\omega}=1 \times 10^{5}$ and $4 \times 10^{5}$, and a second-order accurate FVM and dynamic Smagorinsky sub-grid scale model was used to solve the Navier-Stokes equations and turbulent stresses. At $R e_{\omega}=1 \times 10^{5}$, the stator boundary is in the transitional turbulent state, and the rotor boundary is mostly in the laminar regime. At around $R e_{\omega}=4 \times 10^{5}$, the stator boundary layer becomes fully turbulent, and the rotor boundary layer is in the transitional turbulent state.

The mean velocity profiles of both smooth-wall simulations have been validated against previous experimental and numerical investigations. A similar approach has been followed to validate turbulence intensity profiles at higher Reynolds numbers. It is confirmed that the rotor boundary layer is populated with 15 Type II spiral arms with orientation angle $\epsilon \approx-18^{\circ}$ to the tangential direction. However, no Type I instability has been observed on the rotor boundary layer. This suggests that the transition could be initiated by the interaction of Type II structures with secondary instabilities, as suggested in the investigation of Faller. ${ }^{45}$

Our simulations delivered satisfactory results for both operational Reynolds numbers and agreed with all previous experimental and numerical investigations. This confirms that second-order accurate FVM is accurate enough to simulate rotor-stator cavities in high Reynolds numbers. The solvers, turbulence models, and boundary conditions present in general purpose CFD libraries, like OpenFOAM, are therefore viable alternatives to the in-house higher-order CFD codes that were primarily used in all previous investigations. General purpose CFD libraries are important elements of a modern research practice because of their quality and wide availability. The second-order accuracy of these methods is a good balance between robustness and accuracy and allows comparatively simple numerical simulations using limited computational resources.

The second part of our study involved the simulation of rough wall rotor-stator cavities, using the parametric force model of Busse and Sandham ${ }^{18}$ to simulate roughness effects within our LES formulation. Appropriate modifications were introduced to the original model, and, for simplicity, the roughness effects were only applied to the rotor boundary layer. In all cases, the mean tangential turbulent boundary layer over the rotor showed an outward shift as roughness effects increase, and the velocity profiles of rough wall boundary layers collapse on to the smooth boundary layer profile after a certain axial distance. The peak values of both radial and tangential turbulent intensities of the rotor boundary decrease due to the roughness force term. Both mean and turbulence intensity profiles of the stator boundary show no significant effects due to the rotor roughness effects. The radial turbulent kinetic energy distributions showed that turbulent kinetic energy at the midsection of the cavity was damped due to roughness effects. However, the distributions suggest that the damping was less significant at the outer walls of the rough wall simulations.

Detailed flow visualisations were performed to understand the changes in flow structures of the cavity under the influence of rotor roughness. The resulting structures were visualised from different viewpoints within the cavity. The side view showed that the upper junction between the stator layer and outer wall is more disturbed in rough wall cavities. This observation could be explained by the radial kinetic energy distributions at the outer walls of the cavity. The instantaneous tangential velocity contours at $z^{*}=0.04$ from the rotor plane were extracted, and the rougher cases show intense disturbance behavior. The mean velocity contours preserve the same behavior, but the structures are more sustained in higher $R e_{\omega, \text { local }}$ regions. Similar velocity contours were extracted in a plane near the stator boundary, and, as with the rotor boundary layer, instantaneous tangential velocity contours show intense structures near the outer wall regions. The mean tangential 
velocity contours show that the disturbances are propagating inward from the outer walls of the cavity. These observations suggest that the rough wall rotors have enhanced disturbance propagation, and the bulk fluid streams at the end walls spread these disturbances toward the downstream of the cavity. The high $R e_{\omega}$ cases resulted in iso-surface visualisations near the rotor boundary with enlarged vortex structures on rough wall layers, but did not otherwise show any significant effects on turbulent transition.

The current investigation successfully validated LES simulations of a rotor-stator cavity using a general purpose second order accurate solver. The parametric force approach is deemed to be a plausible method to model roughness effects in DNS and LES methods. However, the current investigation only previews the potential of the parametric model by selecting a few test cases under certain model constants. In future, the model constants could be calibrated to represent more realistic real-world roughness conditions by empirical or direct simulation methods. More test cases can be accommodated to simulate different roughness shape functions, and eventually this will allow the construction of a suitable mapping of parameters between other roughness models, such as, for example, the partial slip model of Miklavčič and Wang. ${ }^{48}$

\section{ACKNOWLEDGMENTS}

This research used the ALICE High Performance Computing Facility at the University of Leicester, UK.

S.J.G. wishes to acknowledge the hospitality of Dr. Sharon Stephen and the Department of Mathematics and Statistics, Faculty of Science at the University of Sydney, Australia where some of this paper was drafted.

\section{NOMENCLATURE}

$\alpha \quad$ roughness factor

$\beta_{1} \quad$ proportionality constant

$\kappa \quad$ von Kármán constant

$v \quad$ fluid kinematic viscosity

$\omega \quad$ disk rotation rate

$\rho \quad$ fluid density

$\sqrt{R_{\theta \theta}} \quad$ tangential turbulence intensity

$\sqrt{R_{r r}} \quad$ radial turbulence intensity

$\tau$

$\tau_{\text {sgs }}$

$\mathrm{Co}$

$F_{B}$

G

$h$

$h_{r}$

K

$k$

$k_{r}$

$k_{\text {disk }}$

$k_{\text {sgs }}$

$P$

sub-grid scale stresses

Courant number

body forces

aspect ratio of the cavity

height of the cavity

roughness height

entrainment coefficient

turbulent kinetic energy

physical roughness height

kinetic energy at the disk surface

sub-grid scale kinetic energy

static pressure

local radial distance to a given point $p$

radius of the rotor hub $r_{2} \quad$ radius of the cavity

$R_{m} \quad$ curvature of the cavity

$R e_{h} \quad$ Reynolds number based on cavity height

$R e_{\omega, \text { local }}$ local Reynolds number

$R e_{\omega} \quad$ Reynolds number based on rotation rate

$u \quad$ instantaneous velocity

$u_{\tau} \quad$ friction velocity

$U_{\theta, \max }$ maximum tangential velocity on the disk

$U_{\theta} \quad$ mean tangential velocity

$U_{r} \quad$ mean radial velocity

$z \quad$ axial distance

${ }^{1}$ V. W. Ekman, "On the influence of the earth's rotation on ocean-currents," Ark. Mat. Astr. Fys. 2(11), 1-53 (1905).

${ }^{2}$ U. T. Bödewadt, "Die drehstr ömung über festem grunde," ZAMM-Z. Angew. Math. Mech. 20, 241-253 (1940).

${ }^{3}$ D. Dijkstra and G. Van Heijst, "The flow between two finite rotating disks enclosed by a cylinder," J. Fluid Mech. 128, 123-154 (1983).

${ }^{4}$ J. W. Daily and R. E. Nece, "Chamber dimension effects on induced flow and frictional resistance of enclosed rotating disks," J. Basic Eng. 82, 217-230 (1960).

${ }^{5}$ A. Sirivat, "Stability experiment of flow between a stationary and a rotating disk," Phys. Fluids A 3, 2664-2671 (1991).

${ }^{6} \mathrm{~N}$. Itoh, "Simple cases of the streamline-curvature instability in threedimensional boundary layers," J. Fluid Mech. 317, 129-154 (1996).

${ }^{7}$ G. Gauthier, P. Gondret, and M. Rabaud, "Axisymmetric propagating vortices in the flow between a stationary and a rotating disk enclosed by a cylinder," J. Fluid Mech. 386, 105-126 (1999).

${ }^{8}$ L. Schouveiler, P. Le Gal, and M. Chauve, "Instabilities of the flow between a rotating and a stationary disk," J. Fluid Mech. 443, 329-350 (2001).

${ }^{9}$ E. Serre, E. C. Del Arco, and P. Bontoux, "Annular and spiral patterns in flows between rotating and stationary discs," J. Fluid Mech. 434, 65-100 (2001).

${ }^{10}$ É. Séverac, S. Poncet, É. Serre, and M.-P. Chauve, "Large eddy simulation and measurements of turbulent enclosed rotor-stator flows," Phys. Fluids 19, 085-113 (2007).

${ }^{11}$ S. Makino, M. Inagaki, and M. Nakagawa, "Laminar-turbulence transition over the rotor disk in an enclosed rotor-stator cavity," Flow, Turbul. Combust. 95, 399-413 (2015).

${ }^{12}$ F. Zoueshtiagh, R. Ali, A. Colley, P. J. Thomas, and P. W. Carpenter, "Laminar-turbulent boundary-layer transition over a rough rotating disk," Phys. Fluids 15, 2441-2444 (2003).

${ }^{13}$ J. Harris, P. Thomas, and S. Garrett, "On the stability of flows over rough rotating disks," in 42nd AIAA Fluid Dynamics Conference and Exhibit (American Institute of Aeronautics and Astronautics, 2012).

${ }^{14}$ A. Cooper, J. Harris, S. J. Garrett, M. Özkan, and P. Thomas, "The effect of anisotropic and isotropic roughness on the convective stability of the rotating disk boundary layer," Phys. Fluids 27, 014107 (2015).

${ }^{15} \mathrm{~S}$. J. Garrett, A. Cooper, J. Harris, M. Özkan, A. Segalini, and P. Thomas, "On the stability of von Kármán rotating-disk boundary layers with radial anisotropic surface roughness," Phys. Fluids 28, 014104 (2016).

${ }^{16}$ B. Alveroglu, A. Segalini, and S. J. Garrett, "The effect of surface roughness on the convective instability of the $B E K$ family of boundary-layer flows," Eur. J. Mech.: B/Fluids 56, 178-187 (2016).

${ }^{17}$ M. Özkan, P. J. Thomas, A. Cooper, and S. J. Garrett, "Comparison of the effects of surface roughness and confinement on rotor-stator cavity flow," Eng. Appl. Comput. Fluid Mech. 11, 142-158 (2017).

${ }^{18}$ A. Busse and N. D. Sandham, "Parametric forcing approach to rough-wall turbulent channel flow," J. Fluid Mech. 712, 169-202 (2012).

${ }^{19}$ F. Moukalled, L. Mangani, M. Darwish et al., The Finite Volume Method in Computational Fluid Dynamics (Springer, 2016).

${ }^{20} \mathrm{R}$. I. Issa, "Solution of the implicitly discretised fluid flow equations by operator-splitting," J. Comput. Phys. 62, 40-65 (1986).

${ }^{21}$ J. Smagorinsky, "General circulation experiments with the primitive equations: I. The basic experiment," Mon. Weather Rev. 91, 99-164 (1963).

${ }^{22}$ M. Germano, U. Piomelli, P. Moin, and W. H. Cabot, "A dynamic subgridscale eddy viscosity model," Phys. Fluids A 3, 1760-1765 (1991).

${ }^{23}$ C. Fureby, G. Tabor, H. Weller, and A. Gosman, "A comparative study of subgrid scale models in homogeneous isotropic turbulence," Phys. Fluids 9, 1416-1429 (1997). 
${ }^{24}$ M. Inagaki, T. Kondoh, and Y. Nagano, "A mixed-time-scale SGS model with fixed model-parameters for practical LES," J. Fluids Eng. 127, 1-13 (2005).

${ }^{25}$ C. S. Peskin, "Numerical analysis of blood flow in the heart," J. Comput. Phys. 25, 220-252 (1977).

${ }^{26} \mathrm{H}$. Choi, P. Moin, and J. Kim, "Direct numerical simulation of turbulent flow over riblets," J. Fluid Mech. 255, 503-539 (1993).

${ }^{27}$ V. De Angelis, P. Lombardi, and S. Banerjee, "Direct numerical simulation of turbulent flow over a wavy wall," Phys. Fluids 9, 2429-2442 (1997).

${ }^{28}$ S. Leonardi and I. P. Castro, "Channel flow over large cube roughness: A direct numerical simulation study," J. Fluid Mech. 651, 519-539 (2010).

${ }^{29} \mathrm{~W}$. Breugem and B. Boersma, "Direct numerical simulations of turbulent flow over a permeable wall using a direct and a continuum approach," Phys. Fluids 17, 025103 (2005).

${ }^{30} \mathrm{~K}$. Bhaganagar, J. Kim, and G. Coleman, "Effect of roughness on wallbounded turbulence," Flow, Turbul. Combust. 72, 463-492 (2004).

${ }^{31}$ R. Taylor, H. Coleman, and B. Hodge, "Prediction of turbulent rough-wall skin friction using a discrete element approach," J. Fluids Eng. 107, 251-257 (1985).

${ }^{32}$ E. Tuck and A. Kouzoubov, "A laminar roughness boundary condition," J. Fluid Mech. 300, 59-70 (1995).

${ }^{33} \mathrm{O}$. Flores and J. Jimenez, "Effect of wall-boundary disturbances on turbulent channel flows," J. Fluid Mech. 566, 357-376 (2006).

${ }^{34}$ J. Cui, V. C. Patel, and C.-L. Lin, "Prediction of turbulent flow over rough surfaces using a force field in large eddy simulation," J. Fluids Eng. 125, 2-9 (2003).

${ }^{35}$ A. Scotti, "Direct numerical simulation of turbulent channel flows with boundary roughened with virtual sandpaper," Phys. Fluids 18, 031701 (2006).
${ }^{36}$ D. Chung, L. Chan, M. MacDonald, N. Hutchins, and A. Ooi, "A fast direct numerical simulation method for characterising hydraulic roughness," J. Fluid Mech. 773, 418-431 (2015).

${ }^{37}$ T. Ishida, G. Brethouwer, Y. Duguet, and T. Tsukahara, "Laminar-turbulent patterns with rough walls," Phys. Rev. Fluids 2, 073901 (2017).

${ }^{38}$ J. Jimenez, "Turbulent flows over rough walls," Annu. Rev. Fluid Mech. 36, 173-196 (2004).

${ }^{39} \mathrm{C}$. Speziale, "Turbulence modeling for time-dependent RANS and VLES: A review," AIAA J. 36, 173-184 (1998).

${ }^{40} \mathrm{~S}$. Cheah, H. Iacovides, D. Jackson, H. Ji, and B. Launder, "Experimental investigation of enclosed rotor-stator disk flows," Exp. Therm. Fluid Sci. 9, 445-455 (1994).

${ }^{41}$ S. B. Pope, Turbulent Flows (Cambridge University Press, 2000).

${ }^{42}$ H. Schlichting, K. Gersten, E. Krause, H. Oertel, and K. Mayes, BoundaryLayer Theory (Springer, 1955), Vol. 7.

${ }^{43}$ M. Itoh, Y. Yamada, S. Imao, and M. Gonda, "Experiments on turbulent flow due to an enclosed rotating disk," Exp. Therm. Fluid Sci. 5, 359-368 (1992).

${ }^{44}$ J. Jeong and F. Hussain, "On the identification of a vortex," J. Fluid Mech. 285, 69-94 (1995).

${ }^{45}$ A. J. Faller, "Instability and transition of disturbed flow over a rotating disk," J. Fluid Mech. 230, 245-269 (1991).

${ }^{46}$ P.-Å. Krogstad, H. Andersson, O. Bakken, and A. Ashrafian, "An experimental and numerical study of channel flow with rough walls," J. Fluid Mech. 530, 327-352 (2005).

${ }^{47}$ P. Orlandi, S. Leonardi, R. Tuzi, and R. Antonia, "Direct numerical simulation of turbulent channel flow with wall velocity disturbances," Phys. Fluids 15, 3587-3601 (2003).

${ }^{48}$ M. Miklavčič and C. Wang, "The flow due to a rough rotating disk," Z. Angew. Math. Phys. (ZAMP) 55, 235-246 (2004). 